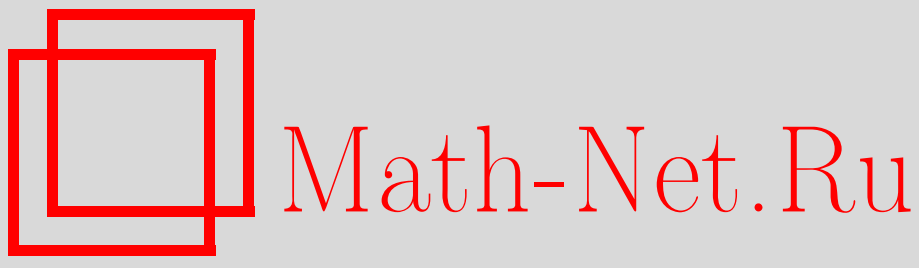

А. Ю. Анфиногенов, И. К. Лифанов, П. И. Лифанов, О некоторых гиперсингулярных одномерных и двумерных интегральных уравнениях, Матем. сб., 2001, том 192, номер 8, 3-46

DOI: https://doi.org/10.4213/sm584

Использование Общероссийского математического портала Math-Net.Ru подразумевает, что вы прочитали и согласны с пользовательским соглашением

http://www.mathnet.ru/rus/agreement

Параметры загрузки:

IP : 3.95.254.165

26 апреля 2023 г., 17:54:18 
А.Ю. Анфиногенов, И.К. Лифанов, П.И. Лифанов

\title{
О некоторых гиперсингулярных одномерных и двумерных интегральных уравнениях
}

\begin{abstract}
Для двумерных сингулярных и гиперсингулярных интегралов даны более общие, чем традиционные, определения. Для гиперсингулярного интеграла на сфере получено новое спектральное соотношение. Рассмотрены квадратурные формулы типа дискретных вихревых пар для одномерных и дискретных замкнутых вихревых рамок для двумерных гиперсингулярных интегралов, изучены вопросы их сходимости, а также сходимости численных решений соответствующих гиперсингулярных интегральных уравнений на отрезке и окружности. Проведен численный эксперимент численного решения гиперсингулярного интегрального уравнения на сфере, из которого видна аналогия численного решения гиперсингулярного интегралњного уравнения на отрезке и сфере.

Библиограффия: 26 названий.
\end{abstract}

\section{Введение}

Теория сингулярных интегральных уравнений, как указано в [1], начала развиваться почти непосредственно вслед за возникновением классической теории интегральных уравнений фредгольма в работах А. Пуанкаре [2] и Д. Гильберта [2], [3]. Однако долгое время теория находилась в тени и только необходимость исследования плоских задач теории упругости игидродинамики вызвала бурное ее развитие, которое было подытожено в двух фундаментальных монографиях [1], [4]. Параллельно задачи практики привели к необходимости численного решения сингулярных интегральных уравнений.

Наиболее ранней работой (1932г.) в этом направлении является работа М.А. Лаврентьева [5], в которой дано численное решение сингулярного интегрального уравнения для обтекания тонкого профиля, основанное на полигональной аппроксимации решения, предвосхитившая в частном случае обшую схему методов типа Ритца-Галёркина. В 1938 году появилась работа Мультхоппа [6], в которой решение одномерного сингулярного интегрального уравнения на отрезке представляется в виде произведения гладкой функции и весовой функции, определяюшей особенности решения на концах. Такая функция раскладьвается в ряд по полиномам Чебьшёва первого рода, а правая часть уравнения раскладывается в ряд по полиномам Чебьшёва второго рода. С помощью спектрального соотношения для соответствуюшего сингулярного оператора между этими полиномами составляется система линейных алгебраических уравнений для коэффициентов этого ряда. Однако такой подход не удалось распространить на двумерные сингулярные интегральные уравнения, к которым сводится пространственная задача обтекания поверхностей. 
В начале пятидесятых годов С.М. Белоцерковский предложил метод дискретных вихрей численного решения одномерных и двумерных сингулярных интегральных уравнений указанного выше типа [7]. С математической точки зрения этот метод является методом коллокации численного решения сингулярных интегральных уравнений на основе применения специальных квадратурных формул типа прямоугольников к соответствующим сингулярньм интегралам.

С другой стороны, сингулярные интегральные уравнения, к которьм сводятся задачи обтекания поверхностей идеальной несжимаемой жидкостью, являются граничньми интегральными уравнениями для решения задачи Неймана для уравнения Лапласа при ее решении с помощью потенциала двойного слоя. В этом случае неизвестной функцией является градиент плотности потенциала двойного слоя, и поэтому в пространственных задачах он имеет две составляющие. Так как эти составляющие не являются независимыми функциями, то получающиеся алгоритмы численного решения пространственных задач обтекания методом дискретных вихревых отрезков имели очень сложную структуру даже для простых поверхностей, лежащих в плоскости [8]. Поэтому в работе [9] было предложено в качестве неизвестной функции брать плотность потенциала двойного слоя и сводить задачу Неймана для уравнения Лапласа к гиперсингулярному интегральному уравнению относительно этой плотности. Решение этого гиперсингулярного интегрального уравнения с помощью специальных квадратурных формул типа прямоугольников получило название метода дискретных замкнутых вихревых рамок [10]. Этот метод быстро получил широкое распространение в задачах аэродинамики, часть которых была опубликована в книге [11]. Параллельно численные методы решения одномерных сингулярных интегральных уравнений начали широко использоваться в различных приложениях (см. [12]-[14] и цитируемую там литературу).

Отметим, что метод дискретных замкнутых вихревых рамок в основном развивался на основе численного эксперимента и физических представлений. Долгое время единственньм математическим результатом в этом направлении был результат И.К. Лифанова о сходимости квадратурных формул типа метода дискретных замкнутых вихревых рамок внутри области с кусочно гладкой гранищей, лежащей в плоскости [15]. Затем в работах Л.Н. Полтавского и И.К. Лифанова вопросы сходимости квадратурных формул и численного решения гиперсингулярных интегральных уравнений были изучены для разомкнутых гладких поверхностей [16], [17]. Для замкнутых гладких поверхностей таких результатов до настоящего времени не было. Численный эксперимент показывал, что численное решение гиперсингулярного интегрального уравнения на сфере равномерно по расчетным точкам сходится к точному [9], [11]. Доказать математически это не удавалось, и было впечатление, что и сами квадратурные формулы для гиперсингулярного интегрального уравнения на сфере равномерно сходятся по расчетным точкам к точному значению интеграла. Этот факт математически доказать не удавалось, в связи с чем А. Ю. Анфиногеновым и П. И. Лифановым был поставлен аккуратный численный эксперимент, в результате которого выяснилось, что поведение квадратурных формул типа дискретных замкнутых вихревых рамок и численного решения соответствуюшего гиперсингулярного интегрального уравнения на сфере подобно поведению аналогичных квадратурных формул и численного решения соответствуюшего гиперсингулярного интегрального уравнения на отрезке. 
В предлагаемой работе доказывается, что квадратурные формулы типа метода дискретных вихревых пар на отрезке (аналог метода дискретных замкнутых вихревых рамок на поверхности) сходятся равномерно по всем расчетным точкам вне фиксированной окрестности концов отрезка и интегрально сходятся на всем отрезке. Численное решение метода дискретных вихревых пар для гиперсингулярного интегрального уравнения на отрезке сходится равномерно на всем отрезке, а разностная производная этого численного решения сходится к производной решения исходного гиперсингулярного интегрального уравнения на отрезке таким образом, каким квадратурные формулы этого метода сходятся к точному значению гиперсингулярного интеграла на отрезке.

Для гиперсингулярного интегрального уравнения типа Гильберта доказано, что квадратурные формулы типа метода дискретных вихревых пар, а также его численное решение этим методом и разностная производная этого численного решения равномерно по расчетным точкам сходятся соответственно к точному значению интеграла, точному решению уравнения и производной этого решения.

Для гиперсингулярного интеграла на сфере доказана равномерная вне полюсов сходимость квадратурных формул метода дискретных замкнутых вихревых рамок. В численном эксперименте показано, что имеется интегральная сходимость этих квадратур на всей сфере и равномерная по всем расчетным точкам сходимость численного решения гиперсингулярного интегрального уравнения на сфере.

В процессе этого доказательства потребовалось дать более общее, чем традиционное, определение двумерного сингулярного интеграла для области на плоскости. Для таких сингулярных интегралов на плоской области предложены некоторые квадратурные формулы типа прямоугольников и доказана их сходимость, если плотности интегралов являются функциями Гёльдера на области интегрирования. Для гиперсингулярного интеграла на сфере получено новое спектральное соотношение для сферических функций. Для гиперсингулярного интеграла на торе доказана равномерная по расчетным точкам сходимость квадратурных формул типа метода дискретных замкнутых вихревых рамок к значению интеграла.

§1. Гиперсингулярные интегралы и уравнения с ними на отрезке и окружности

Рассмотрим вначале следующий интеграл:

$$
I\left(x_{0}\right)=\int_{a}^{b} \frac{d x}{\left(x_{0}-x\right)^{2}}, \quad x_{0} \in(a, b) .
$$

Если определить его по аналогии с интегралом Коши, то получится (при $\varepsilon>0)$ :

$$
\begin{aligned}
I\left(x_{0}\right) & =\lim _{\varepsilon \rightarrow 0}\left(\int_{a}^{x_{0}-\varepsilon} \frac{d x}{\left(x_{0}-x\right)^{2}}+\int_{x_{0}+\varepsilon}^{b} \frac{d x}{\left(x_{0}-x\right)^{2}}\right) \\
& =\lim _{\varepsilon \rightarrow 0}\left(\left.\frac{1}{x_{0}-x}\right|_{a} ^{x_{0}-\varepsilon}+\left.\frac{1}{x_{0}-x}\right|_{x_{0}+\varepsilon} ^{b}\right)=\lim _{\varepsilon \rightarrow 0}\left(\frac{1}{\varepsilon}-\frac{1}{x_{0}-a}+\frac{1}{x_{0}-b}+\frac{1}{\varepsilon}\right) \\
& =\lim _{\varepsilon \rightarrow 0}\left(\frac{2}{\varepsilon}+\frac{1}{x_{0}-b}-\frac{1}{x_{0}-a}\right)
\end{aligned}
$$


Из формулы (1.2) видно, что при $\varepsilon \rightarrow 0$ этот предел равен $+\infty$.

Поэтому, используя идею Адамара [18] о понятии интеграла в смысле конечной части, определим интеграл (1.1) следующим образом:

$$
I\left(x_{0}\right)=\lim _{\varepsilon \rightarrow 0}\left(\int_{I \backslash O\left(x_{0}, \varepsilon\right)} \frac{d x}{\left(x_{0}-x\right)^{2}}-\frac{2}{\varepsilon}\right)=\frac{1}{x_{0}-b}-\frac{1}{x_{0}-a},
$$

где введены обозначения $I=[a, b], O\left(x_{0}, \varepsilon\right)=\left(x_{0}-\varepsilon, x_{0}+\varepsilon\right)$, согласно которым $I \backslash O\left(x_{0}, \varepsilon\right)=\left[a, x_{0}-\varepsilon\right] \cup\left[x_{0}+\varepsilon, b\right]$.

ОпРЕДЕЛЕНИЕ 1.1. Интеграл $I\left(x_{0}\right)$ в формуле (1.1) определим по формуле (1.3) и будем называть гиперсингулярным интегралом на отрезке.

Рассмотрим теперь интеграл

$$
I\left(x_{0}\right)=\int_{a}^{b} \frac{g(x) d x}{\left(x_{0}-x\right)^{2}}, \quad x_{0} \in(a, b),
$$

где функция $g(x)$ определена на $[a, b]$.

ОПРЕДЕЛЕНИЕ 1.2. Интеграл (1.4) будем понимать в смысле равенства

$$
I\left(x_{0}\right)=\lim _{\varepsilon \rightarrow 0}\left(\int_{I \backslash O\left(x_{0}, \varepsilon\right)} \frac{g(x) d x}{\left(x_{0}-x\right)^{2}}-\frac{2 g\left(x_{0}\right)}{\varepsilon}\right)
$$

и называть гиперсингулярным интегралом на отрезке.

Докажем несколько теорем.

Теорема 1.1. Интеграл (1.5) существует для любой функции $g(x) \in H_{1}(\alpha)$ на отрезке $[a, b]$, т.е. $g^{\prime}(x)$ принадлежит классу Гёльдера $H(\alpha)$ степени $\alpha$ $[1 ; \S 3] н а[a, b]$.

ДокАЗАТЕЛЬСТво. Преобразуем формулу (1.5) следующим образом:

$$
\begin{aligned}
I\left(x_{0}\right)= & \lim _{\varepsilon \rightarrow 0}\left[\int_{I \backslash O\left(x_{0}, \varepsilon\right)} \frac{\left(g(x)-g\left(x_{0}\right)-g^{\prime}\left(x_{0}\right)\left(x-x_{0}\right)\right) d x}{\left(x-x_{0}\right)^{2}}\right. \\
& \left.+g\left(x_{0}\right) \int_{I \backslash O\left(x_{0}, \varepsilon\right)} \frac{d x}{\left(x-x_{0}\right)^{2}}-g^{\prime}\left(x_{0}\right) \int_{I \backslash O\left(x_{0}, \varepsilon\right)} \frac{d x}{x_{0}-x}-\frac{2 g\left(x_{0}\right)}{\varepsilon}\right] \\
= & \lim _{\varepsilon \rightarrow 0}\left[\int_{I \backslash O\left(x_{0}, \varepsilon\right)} \frac{\left(g(x)-g\left(x_{0}\right)-g^{\prime}\left(x_{0}\right)\left(x-x_{0}\right)\right) d x}{\left(x-x_{0}\right)^{2}}\right] \\
& +g\left(x_{0}\right) \lim _{\varepsilon \rightarrow 0}\left[\int_{I \backslash O\left(x_{0}, \varepsilon\right)} \frac{d x}{\left(x-x_{0}\right)^{2}}-\frac{2}{\varepsilon}\right]-g^{\prime}\left(x_{0}\right) \lim _{\varepsilon \rightarrow 0} \int_{I \backslash O\left(x_{0}, \varepsilon\right)} \frac{d x}{x_{0}-x} \\
= & I_{1}\left(x_{0}\right)+g\left(x_{0}\right) I_{2}\left(x_{0}\right)-g^{\prime}\left(x_{0}\right) I_{3}\left(x_{0}\right) .
\end{aligned}
$$

В формуле (1.6) интеграл $I_{1}\left(x_{0}\right)$ существует как несобственньй интеграл, так как $g^{\prime}(x) \in H(\alpha)$, и поэтому

$$
\left|g(x)-g\left(x_{0}\right)-g^{\prime}\left(x_{0}\right)\left(x-x_{0}\right)\right| \leqslant A\left|x-x_{0}\right|^{1+\alpha} .
$$

Интеграл $I_{2}\left(x_{0}\right)$ сушествует в смысле определения 1.1 , а интеграл $I_{3}\left(x_{0}\right)$ - в смысле главного значения по Коши $[1 ; \S 11]$. 
ТЕОРема 1.2. Пусть функиия $g(x)$ принадлежсит классу $H_{1}(\alpha)$ на отрезке $[a, b]$. Тогда для интеграла (1.4) справедлива следующая формула интегрирования по частям:

$$
\int_{a}^{b} \frac{g(x) d x}{\left(x-x_{0}\right)^{2}}=\frac{g(b)}{x_{0}-b}-\frac{g(a)}{x_{0}-a}-\int_{a}^{b} \frac{g^{\prime}(x) d x}{x_{0}-x}
$$

ДокАЗАТЕЛЬство. Выполнив в формуле (1.5) интегрирование по частям, получим

$$
\begin{aligned}
I\left(x_{0}\right)= & \lim _{\varepsilon \rightarrow 0}\left(\left.\frac{g(x)}{x_{0}-x}\right|_{a} ^{x_{0}-\varepsilon}+\left.\frac{g(x)}{x_{0}-x}\right|_{x_{0}+\varepsilon} ^{b}-\int_{I \backslash O\left(x_{0}, \varepsilon\right)} \frac{g^{\prime}(x) d x}{x_{0}-x}-\frac{2 g\left(x_{0}\right)}{\varepsilon}\right) \\
= & \frac{1}{x_{0}-b}-\frac{1}{x_{0}-a} \\
& +\lim _{\varepsilon \rightarrow 0}\left(\frac{g\left(x_{0}-\varepsilon\right)}{\varepsilon}+\frac{g\left(x_{0}+\varepsilon\right)}{\varepsilon}-\frac{2 g\left(x_{0}\right)}{\varepsilon}\right)-\lim _{\varepsilon \rightarrow 0}\left(\int_{I \backslash O\left(x_{0}, \varepsilon\right)} \frac{g^{\prime}(x) d x}{x_{0}-x}\right) \\
= & \frac{1}{x_{0}-b}-\frac{1}{x_{0}-a}+I_{4}\left(x_{0}\right)-I_{5}\left(x_{0}\right) .
\end{aligned}
$$

Интеграл $I_{5}\left(x_{0}\right)$ сушествует в смысле главного значения по Коши. Так как $g(x) \in H_{1}(\alpha)$, то

$\left|g\left(x_{0}+\varepsilon\right)+g\left(x_{0}-\varepsilon\right)-2 g\left(x_{0}\right)\right|=\left|\left[g\left(x_{0}+\varepsilon\right)-g\left(x_{0}\right)\right]-\left[g\left(x_{0}\right)-g\left(x_{0}-\varepsilon\right)\right]\right| \leqslant 2 A \varepsilon^{1+\alpha}$,

и поэтому предел $I_{4}\left(x_{0}\right)$ равен нулю для любого $x \in(a, b)$.

ЗАмЕчАнИЕ 1.1. Если $g(a)=g(b)=0$ и $g(x) \in H_{1}(\alpha)$ на $[a, b]$, то формула $(1.8)$ приобретает вид:

$$
\int_{a}^{b} \frac{g(x) d x}{\left(x-x_{0}\right)^{2}}=-\int_{a}^{b} \frac{g^{\prime}(x) d x}{x_{0}-x}, \quad x_{0} \in(a, b) .
$$

ЗАмЕчАНИЕ 1.2. Формула (1.11) справедлива и в том случае, если $g(a)=$ $g(b)=0$, a $g(x) \in H_{1}^{*}(\alpha)$ на $[a, b]$, т.е. производная $g^{\prime}(x) \in H^{*}(\alpha)$ на $[a, b]$, что означает $[1 ; \S 8]$ :

$$
g^{\prime}(x)=\frac{\psi(x)}{(x-a)^{\nu}(b-x)^{\mu}},
$$

где $\psi(x) \in H$ на $[a, b]$, т.е. является функцией Гёльдера на $[a, b]$ некоторой степени, a $\nu, \mu<1$.

Если функция $g(x)$ удовлетворяет требованиям, указанным в замечании 1.2 , то будем говорить, что $g(x) \in H_{1}^{(0,0), *}$ на $[a, b]$.

Рассмотрим теперь для гиперсингулярного интеграла (1.4) на $[a, b]$ следующую квадратурную сумму. Возьмем на $[a, b]$ точки $x_{k}=a+(k-1) h, h=(b-a) / n$, $k=1, \ldots, n+1$, которые разбивают отрезок $[a, b]$ на $n$ равных отрезков $I_{k}=$ $\left[x_{k}, x_{k+1}\right], k=1, \ldots, n$, и точки $x_{0 k}=x_{k}+\frac{h}{2}, k=1, \ldots, n$. Будем говорить, что множества $E=\left\{x_{k}, k=1, \ldots, n\right\}$ и $E_{0}=\left\{x_{0 k}, k=1, \ldots, n\right\}$ образуют 
каноническое разбиение отрезка $[a, b]$. Заменим теперь интеграл (1.4) в точке $x_{0 j} \in E_{0}$ следующей суммой:

$$
S_{n}\left(x_{0 j}\right)=\sum_{k=1}^{n} g\left(x_{0 k}\right) \int_{I_{k}} \frac{d x}{\left(x_{0 j}-x\right)^{2}}=\sum_{k=1}^{n} g\left(x_{0 k}\right)\left(\frac{1}{x_{0 j}-x_{k+1}}-\frac{1}{x_{0 j}-x_{k}}\right) .
$$

\section{Справедлива следуюшая}

ТЕОрема 1.3. Пусть функиия $g(x) \in H_{1}^{*}(\alpha)$ на $[a, b]$. Пусть множсества точек $E$ и $E_{0}$ образуют каноническое разбиение этого отрезка. Тогда справедливо неравенство

$$
\begin{aligned}
& I_{n}\left(x_{0 j}\right)=\left|I\left(x_{0 j}\right)-S_{n}\left(x_{0 j}\right)\right| \\
& =\left|\int_{a}^{b} \frac{g(x) d x}{\left(x_{0 j}-x\right)^{2}}-\sum_{k=1}^{n} g\left(x_{0 k}\right)\left(\frac{1}{x_{0 j}-x_{k+1}}-\frac{1}{x_{0 j}-x_{k}}\right)\right| \\
& \leqslant \Theta\left(x_{0 j}\right), \quad j=1, \ldots, n
\end{aligned}
$$

где величина $\Theta\left(x_{0 j}\right)$ удовлетворяет неравенствам:

а) для всех точек $x_{0 j} \in[a+\delta, b-\delta]$, где $\delta>0-$ сколь угодно малое число,

$$
\Theta\left(x_{0 j}\right) \leqslant C_{\delta} h^{\lambda_{1}}, \quad 0<\lambda_{1}<1,
$$

б) для всех точек $x_{0 j} \in[a, b]$

$$
\sum_{j=1}^{n} \Theta\left(x_{0 j}\right) h \leqslant C h^{\lambda_{2}}, \quad 0<\lambda_{2}<1
$$

әде $C_{\delta}, C$-некоторые константы, не зависящие от $n$.

ДокАЗАТЕЛьство. Интеграл $I\left(x_{0 j}\right)$ представим по формуле $(1.8)$, а сумму $S_{n}\left(x_{0 j}\right)$ запишем в виде

$$
S_{n}\left(x_{0 j}\right)=-\frac{g\left(x_{01}\right)}{x_{0 j}-a}-\sum_{k=2}^{n} \frac{g\left(x_{0 k}\right)-g\left(x_{0 k-1}\right)}{h} \frac{h}{x_{0 j}-x_{k}}+\frac{g\left(x_{0 n}\right)}{x_{0 j}-b} .
$$

Тогда для $I_{n}\left(x_{0 j}\right)$ можно записать:

$$
\begin{aligned}
I_{n}\left(x_{0 j}\right) \leqslant & \left|\frac{g(b)-g\left(x_{0 n}\right)}{x_{0 j}-b}\right|+\left|\frac{g\left(x_{01}\right)-g(a)}{x_{0 j}-b}\right| \\
& +\left|\int_{a}^{b} \frac{g^{\prime}(x) d x}{x_{0 j}-x}-\sum_{k=2}^{n} \frac{g\left(x_{0 k}\right)-g\left(x_{0 k-1}\right)}{h} \frac{h}{x_{0 j}-x_{k}}\right| \\
= & I_{1, n}\left(x_{0 j}\right)+I_{2, n}\left(x_{0 j}\right)+I_{3, n}\left(x_{0 j}\right) .
\end{aligned}
$$

Теперь непосредственно видно, что $I_{1, n}\left(x_{0 j}\right) \leqslant \Theta_{1}\left(x_{0 j}\right), I_{2, n}\left(x_{0 j}\right) \leqslant \Theta_{2}\left(x_{0 j}\right)$, а из результатов $[15 ;$ гл. $1, \S 3]$ следует, что $I_{3, n}\left(x_{0 j}\right) \leqslant \Theta_{3}\left(x_{0 j}\right)$, где каждая из величин $\Theta_{p}\left(x_{0 j}\right), p=1,2,3$, удовлетворяет неравенствам (1.15) и (1.16). Следовательно, сумма величин $\Theta_{p}\left(x_{0 j}\right), p=1,2,3$, также удовлетворяет этим неравенствам. Теорема 1.3 доказана. 
ЗАмечАниЕ 1.3 . Таким образом, квадратурная сумма $S_{n}\left(x_{0 j}\right)$ сходится к значению интеграла $I\left(x_{0 j}\right)$ следуюшим образом. Эта сходимость равномерна по точкам $x_{0 j}$ вне фиксированных окрестностей концов отрезка $[a, b]$, при подходе точки $x_{0 j}$ к одному из концов эта погрешность может стремиться к $\infty$ при $n \rightarrow \infty$, но в целом имеется интегральная сходимость, т.е. выполняется неравенство (1.16).

Рассмотрим теперь уравнение

$$
\int_{-1}^{1} \frac{g(x) d x}{\left(x_{0}-x\right)^{2}}=f\left(x_{0}\right), \quad x_{0} \in(-1,1) .
$$

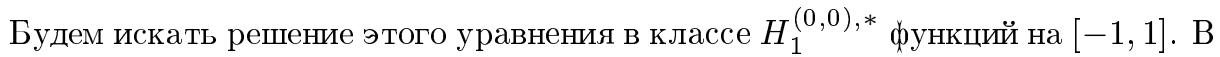
этом классе функций в силу формулы (1.11) уравнение (1.19) эквивалентно уравнению

$$
-\int_{-1}^{1} \frac{g^{\prime}(x) d x}{x_{0}-x}=f\left(x_{0}\right), \quad x_{0} \in(-1,1),
$$

при выполнении условия

$$
\int_{-1}^{1} g^{\prime}(x) d x=g(1)-g(-1)=0
$$

Как следует из [1], решением уравнения (1.20) при условии (1.21) является функция

$$
g^{\prime}(x)=\frac{1}{\pi^{2} \sqrt{1-x^{2}}} \int_{-1}^{1} \frac{\sqrt{1-x_{0}^{2}} f\left(x_{0}\right) d x_{0}}{x-x_{0}}, \quad x \in[-1,1] .
$$

Следовательно, решением уравнения $(1.19)$ из класса $H_{1}^{(0,0)}$,* функций на $[-1,1]$ будет функция

$$
g(x)=\frac{1}{\pi^{2}} \int_{-1}^{x} \frac{d \tau}{\sqrt{1-\tau^{2}}} \int_{-1}^{1} \frac{\sqrt{1-x_{0}^{2}} f\left(x_{0}\right) d x_{0}}{\tau-x_{0}}, \quad x \in[-1,1] .
$$

Так как при последовательном интегрировании можно менять местами порядок интегрирования, если один из интегралов сингулярный, а другой абсолютно интегрируемый $[1 ; \S 28]$, то можно записать:

$$
g(x)=\frac{1}{\pi^{2}} \int_{-1}^{1} \sqrt{1-x_{0}^{2}}\left(\int_{-1}^{x} \frac{d \tau}{\sqrt{1-\tau^{2}\left(\tau-x_{0}\right)}}\right) f\left(x_{0}\right) d x_{0}, \quad x \in[-1,1] .
$$

Непосредственным вычислением с помощью замены переменной $\tau=\cos \varphi$, $0 \leqslant \varphi \leqslant \pi,-1 \leqslant \tau \leqslant 1$, получаем

$$
\int_{-1}^{x} \frac{d \tau}{\sqrt{1-\tau^{2}}\left(\tau-x_{0}\right)}=\frac{1}{\sqrt{1-x_{0}^{2}}} \ln \frac{\left|x_{0}-x\right|}{1-x_{0} x+\sqrt{1-x_{0}^{2}} \sqrt{1-x^{2}}} .
$$

Таким образом, решение уравнения (1.19) для любой функции $f(x) \in H$ на $[-1,1]$ задается формулой

$$
g(x)=\frac{1}{\pi^{2}} \int_{-1}^{1} \ln \frac{\left|x_{0}-x\right|}{1-x_{0} x+\sqrt{1-x_{0}^{2}} \sqrt{1-x^{2}}} f\left(x_{0}\right) d x_{0}, \quad x \in[-1,1] .
$$


Из формулы (1.26) видно, что интеграл в ней имеет логарифмическую особенность на диагонали (при $\left.x=x_{0}\right)$ и $g(-1)=g(1)=0$.

Рассмотрим теперь численное решение гиперсингулярного интегрального уравнения на основе метода дискретных вихревых пар [11; гл. 13, 4 4. Заменим уравнение (1.19) следующей системой линейных алгебраических уравнений, используя каноническое разбиение отрезка $[-1,1]$ :

$$
\sum_{k=1}^{n} g_{n}\left(x_{0 k}\right)\left(\frac{1}{x_{0 j}-x_{k+1}}-\frac{1}{x_{0 j}-x_{k}}\right)=f\left(x_{0 j}\right), \quad j=1, \ldots, n .
$$

Справедлива следуюшая

ТЕОРема 1.4. Пусть функиия $f\left(x_{0}\right)$ принадлежст классу $H$ на отрезке $[-1,1]$. Тогда межсду решением системы линейных алгебраических уравнений (1.27) и решением (1.26) уравнения (1.19) выполняется соотношение

$$
\left|g\left(x_{0 k}\right)-g_{n}\left(x_{0 k}\right)\right| \leqslant C h^{\lambda}, \quad k=1, \ldots, n
$$

где $\lambda>0$ и константа $C$ не зависит от $n$, а между разностной производной $\frac{g_{n}\left(x_{0 k}\right)-g_{n}\left(x_{0 k-1)}\right.}{h} u g^{\prime}\left(x_{k}\right)$ выполняется соотношение

$$
\left|g^{\prime}\left(x_{k}\right)-\frac{g_{n}\left(x_{0 k}\right)-g_{n}\left(x_{0 k-1}\right)}{h}\right| \leqslant \Theta\left(x_{k}\right), \quad k=2, \ldots, n,
$$

где величина $\Theta\left(x_{k}\right), k=2, \ldots, n$, удовлетворяет соотношениям (1.15), (1.16).

ДокАЗАТЕЛьство. Используя представление (1.17), запишем систему (1.27) в эквивалентном виде

$$
\begin{aligned}
-\sum_{k=1}^{n+1} \frac{g_{n}\left(x_{0 k}\right)-g_{n}\left(x_{0 k-1}\right)}{h} \frac{1}{x_{0 j}-x_{k}} & =f\left(x_{0 j}\right), & j=1, \ldots, n, \\
\sum_{k=1}^{n+1} \frac{g_{n}\left(x_{0 k}\right)-g_{n}\left(x_{0 k-1}\right)}{h} h & =0, & j=n+1 .
\end{aligned}
$$

где полагается $g\left(x_{00}\right)=g\left(x_{0 n+1}\right)=0$.

Используя теперь численное решение сингулярного интегрального уравнения, методом дискретных вихрей для индекса $\varkappa=1$ при значении интеграла от решения, равном нулю, получим [15; гл. $5, \S 1]$ :

$$
\frac{g_{n}\left(x_{0 k}\right)-g_{n}\left(x_{0 k-1}\right)}{h}=\frac{1}{h} I_{1, k}^{(n+1)} \sum_{j=1}^{n} \frac{1}{h} I_{1,0 j}^{(n+1)} \frac{f\left(x_{0 j}\right) h}{x_{k}-x_{0 j}}, \quad k=1, \ldots, n+1
$$

где $I_{1, k}^{(n+1)}$ и $I_{1,0 j}^{(n+1)}$ даны в $[15 ;$ гл. $5, \S 1]$ и оттуда же следует справедливость соотношения (1.29), а следовательно, и соотношения (1.28).

Так как описанный выше численный метод типа дискретных вихревых пар [19; гл. $17, \S 1]$ для уравнения (1.19) имеет невысокую скорость сходимости, то желательно построить для этого уравнения численный метод, сходимость которого зависит от дифференциальных свойств функции $f(x)$. Для этого покажем, что для интеграла (1.4) выполняются следующие спектральные соотношения. 
ТЕОРЕМа 1.5. Для интеграла (1.4) въпполняются следующие спектральнье соотношения:

$$
\int_{-1}^{1} \frac{\sqrt{1-x^{2}} U_{n}(x) d x}{\left(x-x_{0}\right)^{2}}=-\pi(n+1) U_{n}\left(x_{0}\right), \quad x \in(-1,1)
$$

әде $U_{n}(x)$ - полином Чебъиёва второго рода.

ДокАЗАТЕЛьство. Используя представление $U_{n}(x)$ и равенство

$$
\sin (\arccos x)=\sqrt{1-x^{2}}
$$

можно записать:

$$
\int_{-1}^{1} \frac{\sqrt{1-x^{2}} U_{n}(x) d x}{\left(x-x_{0}\right)^{2}}=\int_{-1}^{1} \frac{\sin ((n+1) \arccos x) d x}{\left(x-x_{0}\right)^{2}} .
$$

Теперь, в силу формулы интегрирования по частям (1.11), так как

$$
\sin ((n+1) \arccos (1))=\sin ((n+1) \arccos (-1))=0,
$$

получаем

$$
\begin{aligned}
\int_{-1}^{1} \frac{\sin ((n+1) \arccos x) d x}{\left(x-x_{0}\right)^{2}} & =(n+1) \int_{-1}^{1} \frac{\cos ((n+1) \arccos x) d x}{\sqrt{1-x^{2}}\left(x_{0}-x\right)} \\
& =(n+1) \int_{-1}^{1} \frac{T_{n+1}(x) d x}{\sqrt{1-x^{2}}\left(x_{0}-x\right)} \\
& =-\pi(n+1) U_{n}\left(x_{0}\right), \quad x \in(-1,1) .
\end{aligned}
$$

Последнее равенство справедливо в силу соответствуюшего спектрального соотношения для сингулярного интеграла на отрезке [19; гл. 14, 1 1].

Рассмотрим теперь интеграл

$$
I_{\Gamma}\left(\theta_{0}\right)=\int_{0}^{2 \pi} \frac{g(\theta) d \theta}{\sin ^{2}\left(\left(\theta_{0}-\theta\right) / 2\right)}, \quad \theta \in[0,2 \pi]
$$

где плотность $g(\theta)-2 \pi$-периодическая функция. Этот интеграл будем называть гиперсингулярным интегралом типа Гильберта или, если надо подчеркнуть геометрическую сущность этого интеграла, гиперсингулярным интегралом на окружности. Последнее название связано с тем, что [19; гл. $4, \S 3]$ краевая задача Неймана для уравнения Лапласа на окружности приводится с помощью потенциала двойного слоя к гиперсингулярному интегральному уравнению с интегралом вида (1.35).

Как и для гиперсингулярного интеграла на отрезке, рассмотрим вначале случай $g(\theta) \equiv 1$ на $[0,2 \pi]$. Тогда в соответствии с формулами $(1.2)$ и (1.3) дадим следуюшее определение. 
ОПРЕДЕЛЕНИЕ 1.3. Интеграл $I_{\Gamma}\left(\theta_{0}\right)$ при $g(\theta) \equiv 1$ на $[0,2 \pi]$ будем понимать в следуюшем смысле:

$$
\begin{aligned}
I_{\Gamma}\left(\theta_{0}\right) & =\int_{0}^{2 \pi} \frac{d \theta}{\sin ^{2}\left(\left(\theta_{0}-\theta\right) / 2\right)}=\lim _{\varepsilon \rightarrow 0}\left[\int_{[0,2 \pi] \backslash O\left(\theta_{0}, \varepsilon\right)} \frac{d \theta}{\left(\left(\theta_{0}-\theta\right) / 2\right)}-2 \operatorname{ctg} \frac{\varepsilon}{2}\right] \\
& =\lim _{\varepsilon \rightarrow 0}\left[\operatorname{ctg} \frac{\varepsilon}{2}-\operatorname{ctg} \frac{\theta_{0}}{2}+\operatorname{ctg} \frac{\theta_{0}-2 \pi}{2}+\operatorname{ctg} \frac{\varepsilon}{2}-2 \operatorname{ctg} \frac{\varepsilon}{2}\right] \\
& =\operatorname{ctg}\left(\frac{\theta_{0}}{2}-\pi\right)-\operatorname{ctg}\left(\frac{\theta_{0}}{2}\right)=0
\end{aligned}
$$

Теперь для интеграла (1.35) по определению будем полагать:

$$
\int_{0}^{2 \pi} \frac{g(\theta) d \theta}{\sin ^{2}\left(\left(\theta_{0}-\theta\right) / 2\right)}=\lim _{\varepsilon \rightarrow 0}\left[\int_{[0,2 \pi] \backslash O\left(\theta_{0}, \varepsilon\right)} \frac{g(\theta) d \theta}{\sin ^{2}\left(\left(\theta_{0}-\theta\right) / 2\right)}-2 g(\theta) \operatorname{ctg} \frac{\varepsilon}{2}\right] .
$$

Аналогично теоремам 1.1 и 1.2 доказываются следующие теоремы.

ТеОРема 1.6. Интеграл (1.37) существует для любой периодической функиии $g(\theta)$, принадлежсащей классу $H_{1}(\alpha)$ на $[0,2 \pi]$.

ТЕОРЕма 1.7. Пусть функиия $g(\theta) 2 \pi$-периодическая и принадлежст классу $H_{1}(\alpha)$ на $[0,2 \pi]$. Тогда для интеграла (1.35) справедлива следующая формула интегрирования по частям:

$$
\int_{0}^{2 \pi} \frac{g(\theta) d \theta}{\sin ^{2}\left(\left(\theta_{0}-\theta\right) / 2\right)}=-2 \int_{0}^{2 \pi} g^{\prime}(\theta) \operatorname{ctg} \frac{\theta_{0}-\theta}{2} d \theta
$$

Обратимся к рассмотрению квадратурных формул для интеграла (1.35). По аналогии с квадратурной формулой для гиперсингулярного интеграла на отрезке поступим следующим образом. Пусть множества $E=\left\{\theta_{k}, k=1, \ldots, n+1\right\}$ и $E_{0}=\left\{\theta_{0 k}, k=1, \ldots, n\right\}$ образуют каноническое разбиение отрезка $[0,2 \pi]$. Тогда заменим интеграл (1.35) в точке $\theta_{0 j} \in E_{0}$ следуюшей суммой:

$$
\begin{aligned}
S_{\Gamma, n}\left(\theta_{0 j}\right) & =\sum_{k=1}^{n} g\left(\theta_{0 k}\right) \int_{\theta_{k}}^{\theta_{k+1}} \frac{d \theta}{\sin ^{2}\left(\left(\theta_{0 j}-\theta\right) / 2\right)} \\
& =2 \sum_{k=1}^{n} g\left(\theta_{0 k}\right)\left[\operatorname{ctg} \frac{\theta_{0 j}-\theta_{k+1}}{2}-\operatorname{ctg} \frac{\theta_{0 j}-\theta_{k}}{2}\right], \quad j=1, \ldots, n .
\end{aligned}
$$

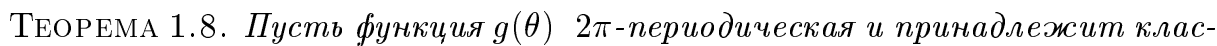
су $H_{1}(\alpha)$ на $[0,2 \pi]$. Пусть множества точек $E$ и $E_{0}$ образуют каноническое разбиение этого отрезка. Тогда справедливо неравенство

$$
\begin{aligned}
& I_{\Gamma, n}\left(\theta_{0 j}\right)=\left|I_{\Gamma}\left(\theta_{0 j}\right)-S_{\Gamma, n}\left(\theta_{0 j}\right)\right|=\mid \int_{0}^{2 \pi} \frac{g(\theta) d \theta}{\sin ^{2}\left(\left(\theta_{0 j}-\theta\right) / 2\right)} \\
& \quad-2 \sum_{k=1}^{n} g\left(\theta_{0 k}\right)\left[\operatorname{ctg} \frac{\theta_{0 j}-\theta_{k+1}}{2}-\operatorname{ctg} \frac{\theta_{0 j}-\theta_{k}}{2}\right] \mid \leqslant C \frac{\ln n}{n^{\alpha}}, \quad j=1, \ldots, n .
\end{aligned}
$$


ДокАзАтЕльство. Интеграл $I_{\Gamma}\left(\theta_{0 j}\right)$ представим по формуле (1.38), а сумму $S_{\Gamma, n}\left(\theta_{0 j}\right)$ запишем в виде

$$
S_{\Gamma, n}\left(\theta_{0 j}\right)=-2 \sum_{k=1}^{n} \frac{g\left(\theta_{0 k}\right)-g\left(\theta_{0 k-1}\right)}{h} \operatorname{ctg} \frac{\theta_{0 j}-\theta_{k}}{2} h, \quad j=1, \ldots, n
$$

где $g\left(\theta_{00}\right)=g\left(\theta_{0 n}\right)$.

Тогда для $I_{\Gamma}\left(\theta_{0 j}\right)$ можно записать

$$
\begin{aligned}
I_{\Gamma}\left(\theta_{0 j}\right) \leqslant 2 & \left|\int_{0}^{2 \pi} g^{\prime}(\theta) \operatorname{ctg} \frac{\theta_{0 j}-\theta}{2} d \theta-\sum_{k=1}^{n} g^{\prime}\left(\theta_{k}\right) \operatorname{ctg} \frac{\theta_{0 j}-\theta_{k}}{2} h\right| \\
& +C_{1} h^{\alpha} \sum_{k=1}^{n}\left|\operatorname{ctg} \frac{\theta_{0 j}-\theta_{k}}{2}\right| h, \quad j=1, \ldots, n .
\end{aligned}
$$

Теперь из результатов [15] для квадратурных формул метода дискретных вихрей для интеграла с ядром Гильберта следует оценка (1.40)

Рассмотрим теперь уравнение

$$
\int_{0}^{2 \pi} \frac{g(\theta) d \theta}{\sin ^{2}\left(\left(\theta_{0}-\theta\right) / 2\right)}=f\left(\theta_{0}\right), \quad \theta_{0} \in[0,2 \pi]
$$

Будем искать решение этого уравнения в классе $H_{1} 2 \pi$-периодических на $[0,2 \pi]$ функций. Сразу заметим, что в силу равенства (1.36) константа является решением однородного уравнения (1.43). Таким образом, решение этого уравнения определено с точностью до константы. Так как функция $\sin ^{-2}\left(\left(\theta_{0}-\theta\right) / 2\right)$ симметрична относительно переменных $\theta_{0}$ и $\theta$, то отсюда и из формулы (1.36) следует, что уравнение (1.43) разрешимо только для тех функций $f(\theta)$, для которых выполняется равенство

$$
\int_{0}^{2 \pi} f(\theta) d \theta=0
$$

Используя теперь формулу (1.38), уравнение (1.43) запишем в виде

$$
-2 \int_{0}^{2 \pi} g^{\prime}(\theta) \operatorname{ctg} \frac{\theta_{0}-\theta}{2} d \theta=f\left(\theta_{0}\right), \quad \theta_{0} \in[0,2 \pi]
$$

Известно $[1 ; \S 33],[19 ;$ гл. $2, \S 4]$, что если $f\left(\theta_{0}\right)$ удовлетворяет условию $(1.44)$ и принадлежит классу $H$ на $[0,2 \pi]$, то решение его $g^{\prime}(\theta)$ тоже принадлежит этому классу, и так как при этом очевидно

$$
\int_{0}^{2 \pi} g^{\prime}(\theta) d \theta=0
$$

в силу $2 \pi$-периодичности функции $g(\theta)$, то получаем

$$
g^{\prime}(\theta)=\frac{1}{8 \pi^{2}} \int_{0}^{2 \pi} \operatorname{ctg} \frac{\theta-\theta_{0}}{2} f\left(\theta_{0}\right) d \theta_{0}, \quad \theta \in[0,2 \pi]
$$


Из формулы (1.47) находим, что

$$
g(\theta)=\frac{1}{4 \pi^{2}} \int_{0}^{2 \pi} \ln \left|\frac{\sin \left(\left(\theta-\theta_{0}\right) / 2\right)}{\sin \left(\theta_{0} / 2\right)}\right| f\left(\theta_{0}\right) d \theta_{0}+C, \quad \theta \in[0,2 \pi]
$$

Так как известно [19], что

$$
\int_{0}^{2 \pi} \ln \left|\sin \left(\left(\theta-\theta_{0}\right) / 2\right)\right| d \theta=-2 \pi \ln 2
$$

то, записав решение (1.48) в виде

$g(\theta)=\frac{1}{4 \pi^{2}} \int_{0}^{2 \pi} \ln \left|\sin \left(\left(\theta-\theta_{0}\right) / 2\right)\right| f\left(\theta_{0}\right) d \theta_{0}+\frac{\ln 2}{4 \pi^{2}} \int_{0}^{2 \pi} f\left(\theta_{0}\right) d \theta_{0}+C, \quad \theta \in[0,2 \pi]$,

получим такую запись решения с произвольной константой $C$, что

$$
\frac{1}{2 \pi} \int_{0}^{2 \pi} g(\theta) d \theta=C
$$

Рассмотрим теперь численное решение уравнения (1.43) опять на основе метода дискретных вихревых пар [11; гл. 17, 4$]$. Заменим уравнение (1.43) следуюшей системой линейных алгебраических уравнений, используя каноническое разбиение отрезка $[0,2 \pi]$ :

$$
\begin{gathered}
\gamma_{0 n}+2 \sum_{k=1}^{n} g_{n}\left(\theta_{0 k}\right)\left(\operatorname{ctg} \frac{\theta_{0 j}-\theta_{k+1}}{2}-\operatorname{ctg} \frac{\theta_{0 j}-\theta_{k}}{2}\right)=f\left(\theta_{0 j}\right), \quad j=1, \ldots, n \\
\frac{1}{2 \pi} \sum_{k=1}^{n} g_{n}\left(\theta_{0 k}\right) \frac{2 \pi}{n}=C
\end{gathered}
$$

где величина $\gamma_{0 n}$ является регуляризующей $[19 ;$ гл. $13, \S 2]$.

Справедлива следуюшая

ТЕОРема 1.9. Пусть функиия $f\left(\theta_{0}\right)$ принадлежсит классу $H(a)$ на $[0,2 \pi]$ и является $2 \pi$-периодической. Тогда мехуду решением системы линейных алгебраических уравнений (1.52) и решением уравнения (1.43), удовлетворяющим условию (1.51), выполняется соотношение

$$
\left|g\left(\theta_{0 k}\right)-g_{n}\left(\theta_{0 k}\right)\right| \leqslant C^{*} n^{-\alpha} \ln n, \quad k=1, \ldots, n
$$

такое же соотношение выполняется между $g^{\prime}\left(\theta_{0 k}\right) u\left[g_{n}\left(\theta_{0 k}\right)-g_{n}\left(\theta_{0 k-1}\right)\right] / h$, $h=2 \pi / n$. 
ДокАЗАтЕльство. Система (1.52) эквивалентна системе

$$
\begin{aligned}
& \gamma_{0 n}-2 \sum_{k=1}^{n} \frac{g_{n}\left(\theta_{0 k}\right)-g_{n}\left(\theta_{0 k-1}\right)}{h} \operatorname{ctg} \frac{\theta_{0 j}-\theta_{k}}{2} h=f\left(\theta_{0 j}\right), \quad j=1, \ldots, n \\
& \sum_{k=1}^{n} \frac{g_{n}\left(\theta_{0 k}\right)-g_{n}\left(\theta_{0 k-1}\right)}{h} h=0 \\
& g_{n}\left(\theta_{00}\right)=g_{n}\left(\theta_{0 n}\right), \\
& \frac{1}{2 \pi} \sum_{k=1}^{n} g_{n}\left(\theta_{0 k}\right) h=C \text {. }
\end{aligned}
$$

Из результатов [19; гл. $13, \S 2]$ следуют разрешимость первых $n+1$ уравнений в системе (1.54) и справедливость неравенства

$$
\left|g^{\prime}\left(\theta_{k}\right)-\frac{g_{n}\left(\theta_{0 k}\right)-g_{n}\left(\theta_{k-1}\right)}{h}\right| \leqslant C_{1} n^{-\alpha} \ln n, \quad k=1, \ldots, n .
$$

Последние два уравнения (1.54) обеспечивают теперь однозначное нахождение величин $g_{n}\left(\theta_{0 k}\right), k=1, \ldots, n$, и совместно с неравенством (1.55) неравенство (1.53). Теорема доказана.

Аналогично доказывается

ТЕОРема 1.10. При условиях теоремы 1.9 на функиию $f(\theta)$ такие жсе соотношения выполняются межсду точным решением уравнения (1.43), удовлетворяющим условию $g\left(\theta_{0 n}\right)=0$, и решением системы линейных алгебраических уравнений

$$
\gamma_{0 n}+2 \sum_{k=1}^{n-1} g_{n}\left(\theta_{0 k}\right)\left(\operatorname{ctg} \frac{\theta_{0 j}-\theta_{k+1}}{2}-\operatorname{ctg} \frac{\theta_{0 j}-\theta_{k}}{2}\right)=f\left(\theta_{0 j}\right), \quad j=1, \ldots, n .
$$

Отметим, что для интеграла (1.35) в [19; гл. $4, \S 3]$ получено следуюшее спектральное соотношение

$$
\begin{aligned}
\int_{0}^{2 \pi} & \frac{1}{\sin ^{2}\left(\left(\theta_{0}-\theta\right) / 2\right)}\left(a_{n} \cos (n \theta)+b_{n} \sin (n \theta)\right) d \theta \\
& =-4 \pi n\left(a_{n} \cos \left(n \theta_{0}\right)+b_{n} \sin \left(n \theta_{0}\right)\right), \quad n=0,1,2, \ldots, \quad \theta_{0} \in[0,2 \pi]
\end{aligned}
$$

где $a_{n}$ и $b_{n}-$ произвольные действительные числа.

\section{§2. Гиперсингулярные интегралы и уравнение с ними на сфере}

Вначале напомним $[19 ;$ гл. $5, \S 3]$ сведение задачи Неймана для уравнения Лапласа к гиперсингулярному интегральному уравнению. Итак, пусть требуется найти функцию $U(M), M(x, y, z) \in E^{3}$, удовлетворяющую уравнению Лапласа

$$
\Delta U(M)=0, \quad M \notin \sigma,
$$


вне поверхности $\sigma$, имеющую заданную нормальную производную на ней

$$
\left.\frac{\partial U}{\partial \bar{n}_{M_{0}}}\right|_{\sigma}=f\left(M_{0}\right), \quad M_{0} \in \sigma
$$

и удовлетворяющую условиям на бесконечности:

$$
|U(M)| \rightarrow 0, \quad|\operatorname{grad} U(M)| \rightarrow 0 \text { при } \rho(M, \sigma) \rightarrow \infty
$$

где $\bar{n}_{M_{0}}-$ орт нормали к поверхности $\sigma$ в точке $M_{0}$,

$$
\operatorname{grad} U(M)=\frac{\partial}{\partial x} U(M) \bar{i}+\frac{\partial}{\partial y} U(M) \bar{j}+\frac{\partial}{\partial z} U(M) \bar{k}
$$

$\bar{i}, \bar{j}, \bar{k}$ - орты осей $O x, O y$ и $O z$ соответственно, а $\rho(M, \sigma)$ - расстояние от точки $M$ до поверхности $\sigma$. Будем предполагать, что поверхность $\sigma$ является гладкой, ограниченной и если она замкнутая, то

$$
\int_{\sigma} f(M) d \sigma=0
$$

Решение задачи (2.1)-(2.3) будем искать в виде потенциала двойного слоя

$$
U\left(M_{0}\right)=\frac{1}{4 \pi} \int_{\sigma} \frac{\partial}{\partial \bar{n}_{M}}\left(\frac{1}{r_{M M_{0}}}\right) g(M) d \sigma_{M},
$$

где $M_{0} \notin \sigma$.

Тогда $[20 ;$ гл. $4, \S 5],[21 ;$ гл. $2, \S 7]$ функция $U\left(M_{0}\right)$ в $(2.5)$ удовлетворяет для точек $M_{0} \notin \sigma$ уравнению (2.1) и соотношению (2.3), а также будет удовлетворять граничному условию (2.2) тогда и только тогда, когда функция $g(M)$ будет решением интегрального уравнения

$$
\frac{1}{4 \pi} \int_{\sigma} \frac{\partial}{\partial \bar{n}_{M_{0}}} \frac{\partial}{\partial \bar{n}_{M}}\left(\frac{1}{r_{M M_{0}}}\right) g(M) d \sigma_{M}=f\left(M_{0}\right), \quad M_{0} \in \sigma .
$$

В уравнении (2.6) знак нормальной производной по точке $M_{0}$ внесен под знак интеграла, так как она непрерывна при переходе точки $M_{0}$ через поверхность $\sigma$ для потенциала двойного слоя [21; гл. $2, \S 5]$.

Выполняя операции дифференцирования, получим

$$
\begin{aligned}
K_{N}\left(M, M_{0}\right) & =\frac{\partial}{\partial \bar{n}_{M_{0}}} \frac{\partial}{\partial \bar{n}_{M}}\left(\frac{1}{r_{M M_{0}}}\right) \\
& =\frac{r_{M M_{0}}^{2}\left(\bar{n}_{M}, \bar{n}_{M_{0}}\right)-3\left(\bar{n}_{M_{0}}, \bar{r}_{M M_{0}}\right)\left(\bar{n}_{M}, \bar{r}_{M M_{0}}\right)}{r_{M M_{0}}^{5}}, \quad M, M_{0} \in \sigma .
\end{aligned}
$$

В частном случае, когда $\sigma$ является частью плоскости $O x y$, получаем

$$
K_{N}\left(M, M_{0}\right)=\frac{1}{r_{M M_{0}}^{3}}
$$


Таким образом, интеграл в (2.6) имеет особенность более высокого порядка, чем двумерный сингулярный интеграл, и его надо понимать в смысле конечного значения по Адамару [18; III, гл. 1, §2]. Дадим его более точное определение в случае, когда $\sigma$ лежит в плоскости $O x y$ и имеет кусочно гладкую границу.

ОПРЕДЕЛЕНИЕ 2.1. Будем полагать

$$
I\left(M_{0}\right)=\frac{1}{4 \pi} \int_{\sigma} \frac{1}{r_{M M_{0}}^{3}} d \sigma_{M}=\frac{1}{4 \pi} \lim _{\varepsilon \rightarrow 0}\left[\int_{\sigma \backslash\left(M_{0}, \varepsilon\right)} \frac{1}{r_{M M_{0}}^{3}} d \sigma_{M}-\frac{2 \pi}{\varepsilon}\right], \quad M_{0} \in \sigma^{-},
$$

где $\sigma^{-}=\sigma \backslash г р \sigma$, где гр $\sigma-$ граница области $\sigma$.

Покажем, что определение 2.1 имеет смысл. Действительно, переходя к полярњым координатам с центром в точке $M_{0}$, получим

$$
\begin{aligned}
I\left(M_{0}\right) & =\frac{1}{4 \pi} \lim _{\varepsilon \rightarrow 0}\left[\int_{0}^{2 \pi} d \varphi \int_{\varepsilon}^{\lambda\left(M_{0}, \varphi\right)} \frac{\rho d \rho}{\rho^{3}}-\frac{2 \pi}{\varepsilon}\right] \\
& =\frac{1}{4 \pi} \lim _{\varepsilon \rightarrow 0}\left[\left.\int_{0}^{2 \pi} d \varphi\left(-\frac{1}{\rho}\right)\right|_{\varepsilon} ^{\lambda\left(M_{0}, \varphi\right)}-\frac{2 \pi}{\varepsilon}\right] \\
& =\frac{1}{4 \pi} \lim _{\varepsilon \rightarrow 0}\left[-\int_{0}^{2 \pi} \frac{d \varphi}{\lambda\left(M_{0}, \varphi\right)}+2 \pi \frac{1}{\varepsilon}-\frac{2 \pi}{\varepsilon}\right] \\
& =-\frac{1}{4 \pi} \int_{0}^{2 \pi} \frac{d \varphi}{\lambda\left(M_{0}, \varphi\right)},
\end{aligned}
$$

где $\rho=\lambda\left(M_{0}, \varphi\right)$ является уравнением границы поверхности $\sigma, M_{0}$ не лежит на гранище поверхности $\sigma$.

Теперь интеграл на части плоскости с произвольной плотностью

$$
I\left(M_{0}\right)=\frac{1}{4 \pi} \int_{\sigma} \frac{g(M) d \sigma_{M}}{r_{M M_{0}}^{3}}, \quad M_{0} \in \sigma
$$

определим следующим образом.

ОПРЕДЕЛЕНИЕ 2.2. Будем полагать

$$
I\left(M_{0}\right)=\frac{1}{4 \pi} \lim _{\varepsilon \rightarrow 0}\left[\int_{\sigma \backslash O\left(M_{0}, \varepsilon\right)} \frac{g(M) d \sigma_{M}}{r_{M M_{0}}^{3}}-\frac{2 \pi g\left(M_{0}\right)}{\varepsilon}\right], \quad M_{0} \in \sigma .
$$

\section{Справедлива следующая}

ТеОрема 2.1. Интеграл (2.12) существует для любой функиии $g(M)$ класса $H_{1}(\alpha)$, т.е. когда $g_{x}^{\prime}$ и $g_{y}^{\prime}$ являются функциями класса $H(\alpha)$ наб. 
ДокАЗАТЕльство. Запишем интеграл (2.12) в виде

$$
\begin{aligned}
I\left(M_{0}\right)= & \frac{1}{4 \pi} \lim _{\varepsilon \rightarrow 0}\left[\int_{\sigma \backslash O\left(M_{0}, \varepsilon\right)} \frac{g(M)-g\left(M_{0}\right)-g_{x}^{\prime}\left(M_{0}\right)\left(x-x_{0}\right)-g_{y}^{\prime}\left(M_{0}\right)\left(y-y_{0}\right)}{r_{M M_{0}}^{3}} d \sigma_{M}\right] \\
& +\frac{1}{4 \pi} g\left(M_{0}\right) \lim _{\varepsilon \rightarrow 0}\left[\int_{\sigma \backslash O\left(M_{0}, \varepsilon\right)} \frac{d \sigma_{M}}{r_{M M_{0}}^{3}}-\frac{2 \pi}{\varepsilon}\right] \\
& +\frac{1}{4 \pi} \lim _{\varepsilon \rightarrow 0}\left[\int_{\sigma \backslash O\left(M_{0}, \varepsilon\right)} \frac{g_{x}^{\prime}\left(M_{0}\right)\left(x-x_{0}\right)+g_{y}^{\prime}\left(M_{0}\right)\left(y-y_{0}\right)}{r_{M M_{0}}^{3}} d \sigma_{M}\right] \\
= & I_{1}\left(M_{0}\right)+I_{2}\left(M_{0}\right)+I_{3}\left(M_{0}\right) .
\end{aligned}
$$

Так как $g(M) \in H_{1}(\alpha)$, то

$$
\left|g(M)-g\left(M_{0}\right)-g_{x}^{\prime}\left(M_{0}\right)\left(x-x_{0}\right)-g_{y}^{\prime}\left(M_{0}\right)\left(y-y_{0}\right)\right| \leqslant C r_{M M_{0}}^{1+\alpha}
$$

и интеграл $I_{1}\left(M_{0}\right)$ существует как несобственньй.

Интеграл $I_{2}\left(M_{0}\right)$ сушествует в смысле определения 2.1.

Интеграл $I_{3}\left(M_{0}\right)$ сушествует как сингулярный интеграл [21; гл. $\left.2, \S 5\right]$, [22; §5].

Пусть теперь $\sigma$ - гладкая поверхность класса $C^{2}[21 ;$ гл. 2, §4], т.е. ее параметрическое представление является дважды непрерывно дифференцируемым на области определения. Тогда величины $\left(\bar{n}_{M_{0}}, \bar{r}_{M M_{0}}\right)$ и $\left(\bar{n}_{M}, \bar{r}_{M M_{0}}\right)$ являются величинами порядка $r_{M M_{0}}^{2}$ при $M \rightarrow M_{0}$. При этих предположениях интеграл по поверхности $\sigma$ от величины $\left(\bar{n}_{M}-\bar{n}_{M_{0}}, \bar{n}_{M_{0}}\right) / r_{M M_{0}}^{3}$ определен как сингулярный двумерный интеграл. Таким образом, для определения интеграла $I_{N}\left(M_{0}\right)$, стоящего в (2.6) слева, достаточно определить интеграл по поверхности $\sigma$ от функции $r_{M M_{0}}^{-3}$, что и сделано в работах [23], [24].

Квадратурные формулы метода дискретных замкнутых вихревых рамок [11; гл. $13, \S 4],[19 ;$ гл. $23, \S 4]$ для интеграла $I_{N}\left(M_{0}\right)$ строятся следующим образом. Пусть поверхность $\sigma$ является образом (достаточно гладким) прямоугольника $\Pi=[a, b] \times[c, d]$ плоскости $O^{*} U V$ при отображении $x=x(U, V), y=y(U, V)$, $z=z(U, V), M^{*}(U, V) \in \Pi$. На каждом отрезке $[a, b]$ и $[c, d]$ возьмем некоторое каноническое разбиение (cм. $\S 1)$ с шагом $h_{1}$ на отрезке $[a, b]$ и шагом $h_{2}$ на отрезке $[c, d]$ так, что $0<q_{1} \leqslant h_{1} / h_{2}, h_{2} / h_{1} \leqslant q_{2}<+\infty$, где $q_{1}$ и $q_{2}-$ некоторые положительные числа. Тогда прямоугольник П разобьется на прямоугольники $\Pi_{k}$ с центрами в точках $M_{0 k}^{*}, k=1, \ldots, n$. Образ прямоугольника $\Pi_{k}$ на поверхности $\sigma$ обозначим $\sigma_{k}$, а точки $M_{0 k}^{*}$ - через $M_{0 k}$. Тогда в точке $M_{0 j}, j=1, \ldots, n$, интеграл $I_{N}\left(M_{0 j}\right)$ заменяется следуюшей суммой:

$$
S_{N, n}\left(M_{0 j}\right)=\sum_{k=1}^{n} g\left(M_{0 k}\right) \int_{\sigma_{k}} K_{N}\left(M, M_{0 j}\right) d \sigma_{M}, \quad j=1, \ldots, n
$$

В частном случае, когда $\sigma$ является прямоугольником П на плоскости $O x y$, в работе [23] доказана 
ТЕОРема 2.2. Пусть функиия $g(x, y)$ представляется в виде

$$
g(x, y)=\sqrt{\rho(x, y)} \Psi(x, y)
$$

где $\rho(x, y)$ - расстояние от точки $M(x, y)$ до границы прямоугольника П, а $\Psi(x, y) \in H_{1}(\alpha), \frac{1}{2} \leqslant \alpha \leqslant 1$, на $\Pi$.

Тогда справедлива оченка

$$
\left|I_{N}\left(M_{0 j}\right)-S_{N, n}\left(M_{0 j}\right)\right|=\Theta_{n}\left(M_{0 j}\right) \leqslant C \frac{h^{1 / 2}}{\rho\left(M_{0 j}\right)}, \quad j=1, \ldots, n
$$

əде $C \geqslant 0$ не зависит от $n$.

Таким образом, из формулы (2.17) видно, что поведение величины $\Theta_{n}\left(M_{0 j}\right)$ аналогично поведению величины $\Theta\left(x_{0 j}\right)$ в формуле $(1.14)$, т.е. $S_{N, n}\left(M_{0 j}\right)$ равномерно сходится к значениям интеграла $I_{N}\left(M_{0 j}\right)$ для точек $M_{0 j}$, отстоящих от границы поверхности не менее, чем на некоторое положительное число, и имеется интегральная сходимость

$$
\sum_{j=1}^{n}\left|I_{N}\left(M_{0 j}\right)-S_{N, n}\left(M_{0 j}\right)\right| s_{\Pi_{j}} \leqslant C_{1} h^{1 / 2} \ln n
$$

где $s_{\Pi_{j}}-$ площадь прямоугольника $\Pi_{j}$.

Рассмотрим теперь более внимательно квадратурные формулы метода дискретных замкнутых вихревых рамок для сферы $C_{R}^{2}$ радиуса $R$. Введем в пространстве $E^{3}$ сферическую систему координат

$$
\left\{\begin{array}{l}
x=r \sin \theta \cos \varphi \\
y=r \sin \theta \sin \varphi \\
z=r \cos \theta
\end{array}\right.
$$

где $r \geqslant 0,0 \leqslant \varphi \leqslant 2 \pi, 0 \leqslant \theta \leqslant \pi$. Тог да уравнение сферы $C_{R}^{2}$ радиуса $R$ с центром в начале координат получается, если в формулах (2.19) положить $r=R$. На отрезке $[0,2 \pi]$ возьмем точки $\varphi_{k}=(k-1) h, h=2 \pi /(2 n)=\pi / n, k=1, \ldots, 2 n+1$, и точки $\varphi_{0 k}=\varphi_{k}+h / 2, k=1, \ldots, 2 n$, и на отрезке $[0, \pi]$ возьмем точки $\theta_{k}=(k-1) h$, $h=\pi / n, k=1, \ldots, n+1$, и $\theta_{0 k}=\theta_{k}+h / 2, k=1, \ldots, n$. Тогда линии $\varphi=\varphi_{i}$, $i=1, \ldots, 2 n+1$, и $\theta=\theta_{k}, k=1, \ldots, n+1$, разобьют прямоугольник $I=[0,2 \pi] \times$ $[0, \pi]$ на прямоугольники $I_{i, k}$ с центрами в точках $M^{*}\left(\varphi_{0 i}, \theta_{0 k}\right), i=1, \ldots, 2 n$, $k=1, \ldots, n$. Образ прямоугольника $I_{i, k}$ на сфере $C_{R}^{2}$ будем обозначать $\sigma_{i, k}$, а образ точки $M^{*}\left(\varphi_{0 i}, \theta_{0 k}\right)$ - через $M_{0 i, 0 k}$. Теперь для интеграла $I_{N}\left(M_{0 j, 0 m}\right)$ на сфере рассмотрим сумму

$$
S_{N, n\left(M_{0 j, 0 m}\right)}=\sum_{i=1}^{2 n} \sum_{k=1}^{n} g\left(M_{0 i, 0 k}\right) \int_{\sigma_{i k}} K_{N}\left(M, M_{0 j, 0 m}\right) d \sigma_{m},
$$

где $j=1, \ldots, 2 n ; m=1, \ldots, n$.

Справедлива следующая 
ТЕОРема 2.3. Пусть функиия $g(M)$ принадлежит классу $H_{1}(\alpha)$ на сфере $C_{R}^{2}$. Тогда для всех точек $M_{0 j, 0 m}$ сферьи таких, что $\theta_{0 m} \in[\delta, \pi-\delta]$, где $\delta>0$ - достаточно малое положстельное число, выполняется оценка

$$
\left|I_{N}\left(M_{0 j, 0 m}\right)-S_{N, n}\left(M_{0 j, 0 m}\right)\right| \leqslant C_{\delta} h^{\alpha} \ln n
$$

Прежде чем доказывать теорему 2.3 , докажем лемму.

Лемма 2.1. Справедливо следующее представление:

$$
\int_{C_{R}^{2}} K_{N}\left(M, M_{0}\right) g(M) d \sigma_{M}=\frac{1}{R} \int_{C_{R=1}^{2}}\left(\frac{1}{r_{M M_{0}}^{3}}+\frac{1}{4 r_{M M_{0}}}\right) g(M) \sin \theta d \theta d \varphi
$$

для любой точки $\left(\varphi_{0}, \theta_{0}\right), 0 \leqslant \varphi_{0} \leqslant 2 \pi, 0 \leqslant \theta_{0} \leqslant \pi$.

ДокАЗАТЕЛьСтво. Для преобразования функции $K_{N}\left(M, M_{0}\right)$ заметим, что для точек сферы $C_{R}^{2}$ справедливы следующие соотношения.

Для величины $r_{M M_{0}}^{2}$ имеем

$$
\begin{aligned}
& r_{M M_{0}}^{2}=R^{2}\left[\left(\sin \theta_{0} \cos \varphi_{0}-\sin \theta \cos \varphi\right)^{2}+\left(\sin \theta_{0} \sin \varphi_{0}-\sin \theta \sin \varphi\right)^{2}\right. \\
& \left.+\left(\cos \theta_{0}-\cos \theta\right)^{2}\right] \\
& =R^{2}\left[\sin ^{2} \theta_{0} \cos ^{2} \varphi_{0}-2 \sin \theta_{0} \sin \theta \cos \varphi_{0} \cos \varphi+\sin ^{2} \theta \cos ^{2} \varphi\right. \\
& +\sin ^{2} \theta_{0} \sin ^{2} \varphi_{0}-2 \sin \theta_{0} \sin \theta \sin \varphi_{0} \sin \varphi+\sin ^{2} \theta \sin ^{2} \varphi \\
& \left.+4 \sin ^{2} \frac{\theta_{0}-\theta}{2} \sin ^{2} \frac{\theta_{0}+\theta}{2}\right] \\
& =R^{2}\left[\sin ^{2} \theta_{0}-2 \sin \theta_{0} \sin \theta \cos \left(\varphi_{0}-\varphi\right)+\sin ^{2} \theta+4 \sin ^{2} \frac{\theta_{0}-\theta}{2} \sin ^{2} \frac{\theta_{0}+\theta}{2}\right] \\
& =R^{2}\left[\left(\sin \theta_{0}-\sin \theta\right)^{2}+2 \sin \theta_{0} \sin \theta\left(1-\cos \left(\varphi_{0}-\varphi\right)\right)\right. \\
& \left.+4 \sin ^{2} \frac{\theta_{0}-\theta}{2} \sin ^{2} \frac{\theta_{0}+\theta}{2}\right] \\
& =R^{2}\left[4 \sin ^{2} \frac{\theta_{0}-\theta}{2} \cos ^{2} \frac{\theta_{0}+\theta}{2}+4 \sin \theta_{0} \sin \theta \sin ^{2} \frac{\varphi_{0}-\varphi}{2}\right. \\
& \left.+4 \sin ^{2} \frac{\theta_{0}-\theta}{2} \sin ^{2} \frac{\theta_{0}+\theta}{2}\right] \\
& =4 R^{2}\left[\sin ^{2} \frac{\theta_{0}-\theta}{2}+\sin \theta_{0} \sin \theta \sin ^{2} \frac{\varphi_{0}-\varphi}{2}\right] \text {. }
\end{aligned}
$$

Далее, из геометрии сферы получаем

$$
\begin{aligned}
\bar{n}_{M} & =\sin \theta \cos \varphi \bar{i}+\sin \theta \sin \varphi \bar{j}+\cos \theta \bar{k} \\
\bar{n}_{M_{0}} & =\sin \theta_{0} \cos \varphi_{0} \bar{i}+\sin \theta_{0} \sin \varphi_{0} \bar{j}+\cos \theta_{0} \bar{k}
\end{aligned}
$$


Из формул (2.24) следует

$$
\begin{aligned}
& \left(\bar{n}_{M}-\bar{n}_{M_{0}}, \bar{n}_{M_{0}}\right)=\left(\sin \theta \cos \varphi-\sin \theta_{0} \cos \varphi_{0}\right) \sin \theta_{0} \cos \varphi_{0} \\
& +\left(\sin \theta \sin \varphi-\sin \theta_{0} \sin \varphi_{0}\right) \sin \theta_{0} \sin \varphi_{0}+\left(\cos \theta-\cos \theta_{0}\right) \cos \theta_{0} \\
& =\sin \theta \cos \varphi \sin \theta_{0} \cos \varphi_{0}-\sin ^{2} \theta_{0} \cos ^{2} \varphi_{0}+\sin \theta \sin \varphi \sin \theta_{0} \sin \varphi_{0} \\
& -\sin ^{2} \theta_{0} \sin ^{2} \varphi_{0}+\cos \theta \cos \theta_{0}-\cos ^{2} \theta_{0} \\
& =\sin \theta \sin \theta_{0} \cos \left(\varphi_{0}-\varphi\right)+\cos \theta \cos \theta_{0}-1 \\
& =\sin \theta \sin \theta_{0}\left[\cos \left(\varphi_{0}-\varphi\right)-1\right]+\cos \theta_{0} \cos \theta+\sin \theta_{0} \sin \theta-1 \\
& =-2 \sin ^{2} \frac{\varphi_{0}-\varphi}{2} \sin \theta \sin \theta_{0}-2 \sin ^{2} \frac{\theta_{0}-\theta}{2} \\
& =-2\left(\sin ^{2} \frac{\theta_{0}-\theta}{2}+\sin \theta \sin \theta_{0} \sin ^{2} \frac{\varphi_{0}-\varphi}{2}\right) \text {. }
\end{aligned}
$$

Из сравнения формул (2.23) и (2.25) получаем

$$
\left(\bar{n}_{M}-\bar{n}_{M_{0}}, \bar{n}_{M_{0}}\right)=-\frac{1}{2 R^{2}} r_{M M_{0}}^{2}
$$

Далее, можно заметить, что

$$
\begin{aligned}
\left(\bar{r}_{M M_{0}}, \bar{n}_{M_{0}}\right)=R\left[\left(\sin \theta_{0} \cos \varphi_{0}-\sin \theta \cos \varphi\right) \sin \theta_{0} \cos \varphi_{0}\right. \\
\left.\quad+\left(\sin \theta_{0} \sin \varphi_{0}-\sin \theta \sin \varphi\right) \sin \theta_{0} \sin \varphi_{0}+\left(\cos \theta_{0}-\cos \theta\right) \cos \theta_{0}\right] \\
=R\left[\sin ^{2} \theta_{0} \cos ^{2} \varphi_{0}-\sin \theta \cos \varphi \sin \theta_{0} \cos \varphi_{0}\right. \\
\left.\quad+\sin ^{2} \theta_{0} \sin ^{2} \varphi_{0}-\sin \theta \sin \varphi \sin \theta_{0} \sin \varphi_{0}+\cos ^{2} \theta_{0}-\cos \theta \cos \theta_{0}\right] \\
=R\left[1-\sin \theta_{0} \sin \theta \cos \left(\varphi_{0}-\varphi\right)-\cos \theta_{0} \cos \theta\right] \\
=R\left[1-\left(\cos \theta_{0} \cos \theta+\sin \theta_{0} \sin \theta\right)+\sin \theta_{0} \sin \theta\left(1-\cos \left(\varphi_{0}-\varphi\right)\right)\right] \\
=2 R\left(\sin ^{2} \frac{\theta_{0}-\theta}{2}+\sin \theta_{0} \sin \theta \sin ^{2} \frac{\varphi_{0}-\varphi}{2}\right)=\frac{1}{2 R} r_{M M_{0}}^{2} .
\end{aligned}
$$

Итак, имеем

$$
\left(\bar{r}_{M M_{0}}, \bar{n}_{M_{0}}\right)=\frac{1}{2 R} r_{M M_{0}}^{2} .
$$

Аналогично получаем

$$
\left(\bar{r}_{M M_{0}}, \bar{n}_{M}\right)=-\left(\bar{r}_{M_{0} M}, \bar{n}_{M}\right)=-\frac{1}{2 R} r_{M M_{0}}^{2} .
$$

Из форомул $(2.7),(2.26),(2.28)$ и (2.29) получаем

$$
\begin{aligned}
\int_{C_{R}^{2}} & K_{N}\left(M, M_{0}\right) g(M) d \sigma_{M} \\
= & \int_{C_{R}^{2}}\left(\frac{1}{r_{M M_{0}}^{3}}-\frac{2}{4 R^{2} r_{M M_{0}}}+\frac{3}{4 R^{2} r_{M M_{0}}}\right) g(M) R^{2} \sin \theta d \theta d \varphi \\
= & \int_{C_{R}^{2}}\left(\frac{1}{r_{M M_{0}}^{3}}+\frac{1}{4 R^{2} r_{M M_{0}}}\right) g(M) R^{2} \sin \theta d \theta d \varphi .
\end{aligned}
$$


Так как для расстояния $r_{R, M M_{0}}$ точек на сфере $C_{R}^{2}$ радиуса $R$ и расстояния $r_{1, \widetilde{M} \widetilde{M}_{0}}$ соответствуюших точек на сфере $C_{R=1}^{2}$ радиуса 1 справедливо соотношение

$$
r_{R, M M_{0}}=R r_{1, \widetilde{M} \widetilde{M}_{0}}
$$

то получаем справедливость леммы 2.1 .

Обратимся теперь к доказательству теоремы 2.3 .

Преобразуем левую часть в неравенстве (2.1) следующим образом:

$$
\begin{aligned}
\left|I_{N}\left(M_{0 j, 0 m}\right)-S_{N, n}\left(M_{0 j, 0 m}\right)\right| & \\
\leqslant & \frac{1}{R}\left|\sum_{i=1}^{2 n} \sum_{k=1}^{n} \int_{\sigma_{i, k}}\left[g(M)-g\left(M_{0 i, 0 k}\right)\right] \frac{\sin \theta d \theta d \varphi}{r_{M M_{0 j, 0 m}}^{3}}\right| \\
& +\frac{1}{R} \sum_{i=1}^{2 n} \sum_{k=1}^{n} \int_{\sigma_{i, k}}\left|g(M)-g\left(M_{0 i, 0 k}\right)\right| \frac{\sin \theta d \theta d \varphi}{r_{M M_{0 j, 0 m}}} \\
= & \frac{1}{R} I_{1}\left(M_{0 j, 0 m}\right)+\frac{1}{R} I_{2}\left(M_{0 j, 0 m}\right), \quad j=1, \ldots, 2 n, \quad m=1, \ldots, n .
\end{aligned}
$$

Вначале оценим величину $I_{2}\left(M_{0 j, 0 m}\right)$. Для этого напомним, что так как $g(M) \in$ $H_{1}(\alpha)$ на сфере $C_{R}^{2}$, то справедливо неравенство

$$
\left|g(M)-g\left(M_{0}\right)\right| \leqslant A r_{M M_{0}}
$$

где $M, M_{0} \in C_{R}^{2}, r_{M M_{0}}$ - расстояние между соответствующими точками на сфере единичного радиуса, $A>0$ - некоторая константа.

Представим теперь $I_{2}\left(M_{0 j, 0 m}\right)$ в виде

$$
\begin{aligned}
I_{2}\left(M_{0 j, 0 m}\right)= & \int_{\sigma_{j, m}} \frac{\left|g(M)-g\left(M_{0 j, 0 m}\right)\right|}{r_{M M_{0 j, 0 m}}} \sin \theta d \theta d \varphi \\
& +\sum_{\substack{i=1 \\
(i, k) \neq(j, m)}}^{2 n} \sum_{\sigma_{j, m}} \frac{\left|g(M)-g\left(M_{0 i, 0 k}\right)\right|}{r_{M M_{0 j, 0 m}}} \sin \theta d \theta d \varphi \\
= & I_{2,1}\left(M_{0 j, 0 m}\right)+I_{2,2}\left(M_{0 j, 0 m}\right) .
\end{aligned}
$$

Для $I_{2,1}\left(M_{0 j, 0 m}\right)$ с учетом $(2.33)$ имеем

$$
I_{2,1}\left(M_{0 j, 0 m}\right) \leqslant A \int_{\sigma_{j, m}} \sin \theta d \theta d \varphi \leqslant A h^{2} .
$$

Для оценки $I_{2,2}\left(M_{0 j, 0 m}\right)$ заметим, что справедливо неравенство (см. рис. 1):

$$
\alpha \geqslant \sin \alpha \geqslant \frac{2}{\pi} \alpha, \quad \alpha \in\left[0, \frac{\pi}{2}\right]
$$




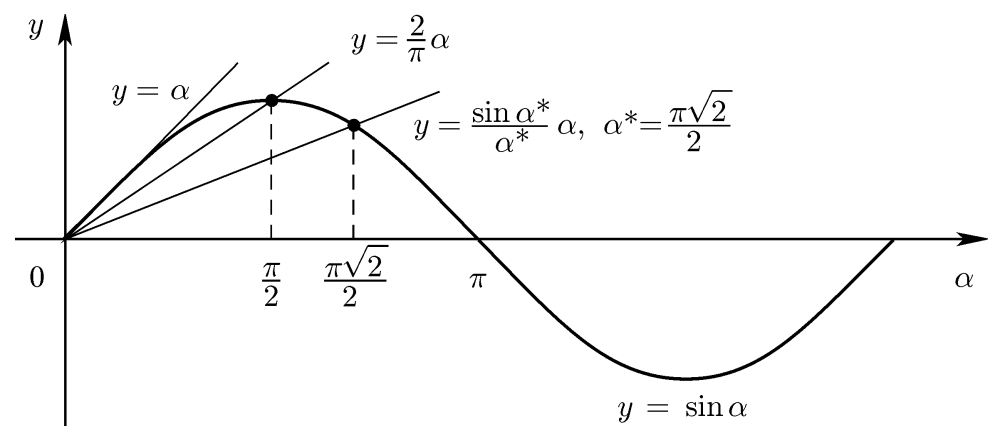

Рис. 1

Поэтому из $(2.23)$ и (2.33) имеем

$$
\begin{aligned}
\left|g(M)-g\left(M_{0 i, 0 k}\right)\right| & \leqslant A r_{M M_{0 i, 0 k}} \leqslant A 2 \sqrt{\sin ^{2} \frac{\theta_{0 k}-\theta}{2}+\sin ^{2} \frac{\varphi_{0 k}-\varphi}{3}} \\
& \leqslant A 2 \sqrt{\frac{\left(\theta_{0 k}-\theta\right)^{2}}{4}+\frac{\left(\varphi_{0 k}-\varphi\right)^{2}}{4}} \\
& =A \sqrt{\left(\theta_{0 k}-\theta\right)^{2}-\left(\varphi_{0 k}-\varphi\right)^{2}} \leqslant A \frac{h}{\sqrt{2}},
\end{aligned}
$$

ког да $M(\varphi, \theta) \in I_{i, k}$.

Введем на прямоугольнике $I=[0,2 \pi] \times[0, \pi]$ переменных $\varphi$ и $\theta$ полярные координаты с центром в точке $M\left(\varphi_{0 j}, \theta_{0 m}\right)$ :

$$
\left\{\begin{array}{l}
\varphi-\varphi_{0 j}=r \cos \alpha, \\
\theta-\theta_{0 m}=r \sin \alpha
\end{array}\right.
$$

где $0 \leqslant \alpha \leqslant 2 \pi, 0 \leqslant r \leqslant \pi \sqrt{2}$. Последние неравенства справедливы, так как в силу периодичности всех функций в рассматриваемом интеграле по $\varphi$ можно полагать, что разность $\left(\varphi-\varphi_{0 j}\right)$ меняется в пределах от $-\pi$ до $\pi$ и, тем более, в этих пределах меняется разность $\left(\theta-\theta_{0 m}\right)$. Теперь в силу формулы $(2.23)$ можно записать для точек единичной сферы:

$$
\begin{aligned}
r_{M M_{0 j, 0 m}}^{2} & =4\left[\sin ^{2} A+\sin \theta_{0} \sin \theta \sin ^{2} B\right] \\
& =4 r^{2}\left[\frac{\sin ^{2} A}{A^{2}} \frac{\cos ^{2} \alpha}{4}+\sin \theta_{0} \sin \theta \frac{\sin ^{2} B}{B^{2}} \frac{\sin ^{2} \alpha}{4}\right],
\end{aligned}
$$

где $A=\frac{1}{2} r \cos \alpha, B=\frac{1}{2} r \sin \alpha$. Теперь в силу ограничения на $r$ (см. (2.38)) получим, что

$$
0 \leqslant\left|r \frac{\cos \alpha}{2}\right|, \quad\left|r \frac{\sin \alpha}{2}\right| \leqslant \frac{\sqrt{2} \pi}{2}=\alpha^{*} ;
$$

и поэтому (см. рис. 1) имеем

$$
\frac{\sin ^{2} \alpha^{*}}{\left(\alpha^{*}\right)^{2}} \leqslant \frac{\sin ^{2} A}{A^{2}}, \quad \frac{\sin ^{2} B}{B^{2}} \leqslant 1 .
$$


Заметим, что для любых положительных чисел $a$ и $b$ выполняется соотношение

$$
\min (a ; b) \leqslant a \cos ^{2} \alpha+b \sin ^{2} \alpha \leqslant \max (a ; b)
$$

для любых $\alpha$. Следовательно, из формул (2.39)-(2.42) получаем

$$
r^{2} \geqslant r_{M M_{0 j, 0 m}}^{2} \geqslant \lambda^{2} r^{2}
$$

для любых $M$ и $M_{0 j, 0 m}$, где $\lambda^{2}=\min \left(\left(\sin \alpha^{*} / \alpha^{*}\right)^{2} ; \sin \theta_{0 m} \sin \theta\right)$, или

$$
1 \geqslant \frac{r_{M M_{0 j, 0 m}}}{r} \geqslant \lambda
$$

Возвращаясь теперь к оценке $I_{2,2}\left(M_{0 j, 0 m}\right)$ с переходом к полярным координатам $(r, \alpha)$, получим

$$
\begin{gathered}
I_{2,2}\left(M_{0 j, 0 m}\right) \leqslant \frac{A}{\sqrt{2}} h \int_{I \backslash I_{j, m}} \frac{\sin \theta d \theta d \varphi}{r \lambda} \leqslant \frac{C_{1} h}{\sqrt{\rho^{*}\left(\theta_{0 m}\right)}} \int_{I \backslash I_{j, m}} \frac{\sqrt{\sin \theta} d \theta d \varphi}{r} \\
\leqslant \frac{C_{1} h}{\sqrt{\rho^{*}\left(\theta_{0 m}\right)}} \int_{0}^{2 \pi} d \alpha \int_{h / 2}^{2 \pi} \frac{r d r}{r}=\frac{C_{1} h}{\sqrt{\rho^{*}\left(\theta_{0 m}\right)}} 4^{2} \pi^{2}=\frac{C_{2}}{\sqrt{\rho^{*}\left(\theta_{0 m}\right)}} h,
\end{gathered}
$$

где $\rho^{*}\left(\theta_{0 m}\right)=\min \left(\theta_{0 m} ; \pi-\theta_{0 m}\right)$.

Обратимся теперь к оценке $I_{1}\left(M_{0 j, 0 m}\right)$. Введем величину $\widetilde{r}_{M M_{0}}$ для точек единичной сферы по формуле

$$
\widetilde{r}_{M M_{0}}=2\left[\sin ^{2} \frac{\theta_{0}-\theta}{2}+\sin ^{2} \theta_{0} \sin ^{2} \frac{\varphi_{0}-\varphi}{2}\right]^{1 / 2} .
$$

Преобразуем выражение для $I_{1}\left(M_{0 j, 0 m}\right)$ следующим образом:

$$
\begin{gathered}
I_{1}\left(M_{0 j, 0 m}\right) \leqslant \mid \sum_{i=1}^{2 n} \sum_{k=1}^{n} \int_{\sigma_{i, k}} \frac{g(M)-g\left(M_{0 i, 0 k}\right)}{\widetilde{r}_{M M_{0 j, 0 m}}^{3} \sin \theta d \theta d \varphi \mid} \\
+\left|\sum_{i=1}^{2 n} \sum_{k=1}^{n} \int_{\sigma_{i, k}}\left[g(M)-g\left(M_{0 i, 0 k}\right)\right]\left[\frac{1}{r_{M M_{0 j, 0 m}}^{3}}-\frac{1}{\widetilde{r}_{M M_{0 j, 0 m}}^{3}}\right] \sin \theta d \theta d \varphi\right| \\
=I_{1,1}\left(M_{0 j, 0 m}\right)+I_{1,2}\left(M_{0 j, 0 m}\right) .
\end{gathered}
$$

Введем обозначения $r_{M M_{0 j, 0 m}}^{2}=c$ и $\widetilde{r}_{M M_{0 j, 0 m}}^{2}=d$. Тогда можно записать

$$
\begin{aligned}
& \frac{1}{r_{M M_{0 j, 0 m}}^{3}}-\frac{1}{\widetilde{r}_{M M_{0 j, 0 m}}^{3}}=\frac{1}{c^{3 / 2}}-\frac{1}{d^{3 / 2}}=\frac{d^{3 / 2}-c^{3 / 2}}{c^{3 / 2} d^{3 / 2}} \\
& =\frac{d^{3}-c^{3}}{c^{3 / 2} d^{3 / 2}\left[d^{3 / 2}+c^{3 / 2}\right]}=\frac{(d-c)\left(d^{2}+d c+c^{2}\right)}{c^{3 / 2} d^{3 / 2}\left(d^{3 / 2}+c^{3 / 2}\right)} \\
& =\frac{d-c}{c^{3 / 2} d}\left[\frac{d^{3 / 2}}{d^{3 / 2}+c^{3 / 2}}+\frac{d^{1 / 2} c^{2 / 2}}{d^{3 / 2}+c^{3 / 2}}\right]+\frac{d-c}{c d^{3 / 2}} \frac{c^{3 / 2}}{d^{3 / 2}+c^{3 / 2}}
\end{aligned}
$$


Заметим, что поскольку величины $d$ и $c$ положительны, то справедливы неравенства

$$
\frac{d^{3 / 2}}{d^{3 / 2}+c^{3 / 2}} \leqslant 1, \quad \frac{d^{1 / 2} c^{2 / 2}}{d^{3 / 2}+c^{3 / 2}} \leqslant 1, \quad \frac{c^{3 / 2}}{d^{3 / 2}+c^{3 / 2}} \leqslant 1
$$

для произвольных точек $M$ и $M_{0 j, 0 m}$.

Запишем теперь разность $d-c$ в виде

$$
\begin{aligned}
d-c & =\widetilde{r}_{M M_{0}}^{2}-r_{M M_{0}}^{2}=4 \sin ^{2} \frac{\varphi_{0}-\varphi}{2} \sin \theta_{0}\left(\sin \theta_{0}-\sin \theta\right) \\
& =8 \sin ^{2} \frac{\varphi_{0}-\varphi}{2} \sin \frac{\theta_{0}-\theta}{2} \cos \frac{\theta_{0}+\theta}{2} \sin \theta_{0}
\end{aligned}
$$

при $M_{0}=M_{0 j, 0 m}$.

Теперь обратимся к слагаемому $I_{1,2}\left(M_{0 j, 0 m}\right)$. Используя формулы $(2.37)$ и (2.46)-(2.49), получим

$$
\begin{aligned}
I_{1,2}\left(M_{0 j, 0 m}\right) \leqslant \int_{\sigma_{j, m}} \frac{8 A \sin ^{2}\left(\left(\varphi_{0 j}-\varphi\right) / 2\right) \sin \left|\left(\theta_{0 m}-\theta\right) / 2\right| \sin \theta_{0 m}}{r_{M} M_{0 j, 0 m} \widetilde{r}_{M M_{0 j, 0 m}}^{2}} \\
\quad \times\left(\frac{2}{r_{M M_{0 j, 0 m}}}+\frac{1}{\widetilde{r}_{M M_{0 j, 0 m}}}\right) \sin \theta d \theta d \varphi \\
\quad+\frac{8 A h}{\sqrt{2}} \int_{C_{R}^{2} \backslash \sigma_{j, m}} \frac{\sin ^{2}\left(\left(\varphi_{0 j}-\varphi\right) / 2\right) \sin \left|\left(\theta_{0 m}-\theta\right) / 2\right| \sin \theta_{0 m}}{\widetilde{r}_{M M_{0 j, 0 m}}^{2}} \\
\quad \times\left(\frac{2}{r_{M M_{0 j, 0 m}}}+\frac{1}{\widetilde{r}_{M M_{0 j, 0 m}}}\right) \sin \theta d \theta d \varphi \\
\quad=I_{1,2}^{*}\left(M_{0 j, 0 m}\right)+I_{1,2}^{* *}\left(M_{0 j, 0 m}\right) .
\end{aligned}
$$

Заметим теперь, что для величины $\widetilde{r}_{M M_{0 j, 0 m}}$ справедливы соотношения

$$
1 \geqslant \frac{\widetilde{r}_{M M_{0 j, 0 m}}}{r} \geqslant \lambda_{1}
$$

где $\lambda_{1}=\min \left(\sin \alpha^{*} / \alpha^{*} ; \sin \theta_{0 m}\right)$ для любых точек $M$ и $M_{0 j, 0 m}$.

Далее, справедливы неравенства

$$
\begin{gathered}
\frac{\sin \left|\left(\theta_{0 m}-\theta\right) / 2\right|}{\widetilde{r}_{M M_{0 j, 0 m}}} \leqslant 1, \quad \frac{\sin \left|\left(\theta_{0 m}-\theta\right) / 2\right|}{r_{M M_{0 j, 0 m}}} \leqslant 1 \\
\frac{\sin ^{2}\left(\left(\varphi_{0 j}-\varphi\right) / 2\right) \sin \theta_{0 m}}{\widetilde{r}_{M M_{0 j, 0 m}}^{2}} \leqslant \frac{1}{\sin \theta_{0 m}}
\end{gathered}
$$

для любых точек $M$ и $M_{0 j, 0 m}$. С учетом неравенств $(2.52),(2.53)$, переходя к полярньм координатам (2.38), имеем

$$
\begin{aligned}
I_{1,2}^{*}\left(M_{0 j, 0 m}\right) & \leqslant 8 A \int_{0}^{2 \pi} \int_{0}^{h / \sqrt{2}} \frac{1}{r \sin \theta_{0 m}}\left(\frac{2}{\lambda}+\frac{1}{\lambda_{1}}\right) \sin \theta r d r d \alpha \\
& \leqslant A_{1} \int_{0}^{2 \pi} d \alpha \int_{0}^{h / \sqrt{2}} \frac{1}{\sin \theta_{0 m}}\left(\frac{2}{\sqrt{\sin \theta_{0 m}}}+\frac{1}{\sin \theta_{0 m}}\right) d r \\
& \leqslant A_{2} h \frac{1}{\sin ^{2} \theta_{0 m}}, \quad j=1, \ldots, 2 n, \quad m=1, \ldots, n .
\end{aligned}
$$


Для $I_{1,2}^{* *}\left(M_{0 j, 0 m}\right)$ имеем аналогично

$$
\begin{aligned}
I_{1,2}^{* *}\left(M_{0 j, 0 m}\right) & \leqslant \frac{8 A h}{\sqrt{2}} \int_{0}^{2 \pi} d \alpha \int_{h / 2}^{2 \pi} \frac{1}{r \lambda} \frac{1}{\sin \theta_{0 m}}\left(\frac{2}{\lambda}+\frac{1}{\lambda_{1}}\right) \frac{\sin \theta r d r}{r} \\
& \leqslant A_{3} h\left(\frac{2}{\left(\sin \theta_{0 m}\right)^{3 / 2}}+\frac{1}{\left(\sin \theta_{0 m}\right)^{5 / 2}}\right) \int_{0}^{2 \pi} d \alpha \int_{h / 2}^{2 \pi} \frac{d r}{r} \\
& \leqslant A_{4} h \frac{1}{\left(\sin ^{2} \theta_{0 m}\right)^{5 / 2}} \ln n, \quad j=1, \ldots, 2 n, \quad m=1, \ldots, n .
\end{aligned}
$$

Таким образом, из формул (2.50) и (2.54), (2.55) получаем

$$
I_{1,2}\left(M_{0 j, 0 m}\right) \leqslant A_{5} \frac{1}{\left(\sin \theta_{0 m}\right)^{5 / 2}} h \ln n .
$$

Теперь рассмотрим оценку для $I_{1,1}\left(M_{0 j, 0 m}\right)$ в $(2.46)$. Запишем $I_{1,1}\left(M_{0 j, 0 m}\right)$ в следуюшем виде:

$$
\begin{aligned}
I_{1,1}\left(M_{0 j, 0 m}\right) \leqslant & \left|\sum_{i=1}^{2 n} \sum_{k=1}^{n} \int_{\sigma_{i, k}} \frac{\Delta\left(M, M_{0 i, 0 k}\right)}{\widetilde{r}_{M M_{0 j, 0 m}}^{3}} \sin \theta d \theta d \varphi\right| \\
& +\left|\sum_{i=1}^{2 n} \sum_{k=1}^{n} \int_{\sigma_{i, k}} \frac{g_{\varphi}^{\prime}(M)\left(\varphi-\varphi_{0 i}\right)}{\widetilde{r}_{M M_{0 j, 0 m}}^{3}} \sin \theta d \theta d \varphi\right| \\
& +\left|\sum_{i=1}^{2 n} \sum_{k=1}^{n} \int_{\sigma_{i, k}} \frac{g_{\theta}^{\prime}(M)\left(\theta-\theta_{0 k}\right)}{\widetilde{r}_{M M_{0 j, 0 m}}^{3}} \sin \theta d \theta d \varphi\right| \\
= & I_{1,1}^{*}\left(M_{0 j, 0 m}\right)+I_{1,1}^{* *}\left(M_{0 j, 0 m}\right)+I_{1,1}^{* *}\left(M_{0 j, 0 m}\right),
\end{aligned}
$$

где $\Delta\left(M, M_{0 i, 0 k}\right)=g(M)-g\left(M_{0 i, 0 k}\right)-g_{\varphi}^{\prime}(M)\left(\varphi-\varphi_{0 i}\right)-g_{\theta}^{\prime}(M)\left(\theta-\theta_{0 k}\right)$.

Для $I_{1,1}^{*}\left(M_{0 j, 0 m}\right)$ с учетом формулы для $\Delta\left(M, M_{0 i, 0 k}\right)$ и того, что $g(M) \in H_{1}(\alpha)$ на сффере, получаем

$$
\begin{aligned}
& I_{1,1}^{*}\left(M_{0 j, 0 m}\right) \leqslant A^{*} \int_{\sigma_{j, m}} \frac{r_{M M_{0 j, 0 m}}^{1+\alpha} \sin \theta d \theta d \varphi+A_{1}^{*} h^{1+\alpha} \int_{C_{R}^{2} \backslash \sigma_{j, m}} \frac{\sin \theta d \theta d \varphi}{\widetilde{r}_{M M_{0 j, 0 m}}^{3}}}{\leqslant A^{*}\left[\int_{\sigma_{j, m}} \frac{r_{M M_{0 j, 0 m}}^{1+\alpha}\left|\sin \theta-\sin \theta_{0 m}\right|}{\widetilde{r}_{M M_{0 j, 0 m}}^{3}} d \theta d \varphi+\sin \theta_{0 m} \int_{\sigma_{j, m}} \frac{r_{M M_{0 j, 0 m}}^{1+\alpha}}{\widetilde{r}_{M M_{0 j, 0 m}}^{3}} d \theta d \varphi\right]} \\
& \quad+A_{1}^{*} h^{1+\alpha}\left[\int_{C_{R}^{2} \backslash \sigma_{j, m}} \frac{\left|\sin \theta-\sin \theta_{0 m}\right|}{\widetilde{r}_{M M_{0 j, 0 m}}^{3}} d \theta d \varphi+\sin \theta_{0 m} \int_{C_{R}^{2} \backslash \sigma_{j, m}} \frac{d \theta d \varphi}{\widetilde{r}_{M M_{0 j, 0 m}}^{3}}\right] .
\end{aligned}
$$

Переходя к полярным координатам (2.38) и проводя выкладки, аналогичные проведенным для оценки $I_{1,2}^{* *}\left(M_{0 j, 0 m}\right)$, получим

$$
I_{1,1}^{*}\left(M_{0 j, 0 m}\right) \leqslant A_{2}^{*} \frac{h^{\alpha}}{\sin ^{2} \theta_{0 m}} .
$$


Для оценки $I_{1,1}^{* *}\left(M_{0 j, 0 m}\right)$ запишем его вначале в виде

$$
\begin{aligned}
I_{1,1}^{* *}\left(M_{0 j, 0 m}\right) \leqslant & \left|\sum_{i=1}^{2 n} \sum_{k=1}^{n} \int_{I_{i, k}} \frac{\left[g_{\varphi}^{\prime}(M)-g_{\varphi}^{\prime}\left(M_{0 i, 0 k}\right)\right]\left(\varphi-\varphi_{0 i}\right)}{\widetilde{r}_{M M_{0 j, 0 m}}^{3}} \sin \theta d \theta d \varphi\right| \\
& +B_{1}\left|\sum_{i=1}^{2 n} \sum_{k=1}^{n} \int_{I_{i, k}} \frac{\left(\varphi-\varphi_{0 i}\right)\left(\sin \theta-\sin \theta_{0 m}\right)}{\widetilde{r}_{M M_{0 j, 0 m}}^{3}} d \theta d \varphi\right| \\
& +B_{1}\left|\sin \theta_{0 m}\right|\left|\sum_{i=1}^{2 n} \sum_{k=1}^{n} \int_{I_{i, k}} \frac{\varphi-\varphi_{0 i}}{\widetilde{r}_{M M_{0 j, 0 m}}^{3}} d \theta d \varphi\right| \\
= & \eta_{1}\left(M_{0 j, 0 m}\right)+B_{1} \eta_{2}\left(M_{0 j, 0 m}\right)+B_{1}\left|\sin \theta_{0 m}\right| \eta_{3}\left(M_{0 j, 0 m}\right),
\end{aligned}
$$

где $B_{1}=\max _{M \in C_{R}^{2}} g_{\varphi}^{\prime}(M)$.

Теперь, записав $\eta_{3}\left(M_{0 j, 0 m}\right)$ в виде

$$
\eta_{3}\left(M_{0 j, 0 m}\right)=\left|\sum_{i=1}^{2 n} \sum_{k=1}^{n} \int_{I_{i, k}} \frac{\varphi-\varphi_{0 i}}{\widetilde{r}_{M M_{0 j, 0 m}}^{3}} d \theta d \varphi+\sum_{i=1}^{2 n} \sum_{k=1}^{n} \int_{I_{i, k}} \frac{\varphi_{0 j}-\varphi_{0 i}}{\widetilde{r}_{M M_{0 j, 0 m}}^{3}} d \theta d \varphi\right|
$$

видим, что $\eta_{3}\left(M_{0 j, 0 m}\right)=0$ в силу нечетности по $\left(\varphi-\varphi_{0 j}\right)$ подынтегральной функции (напомним, что $\varphi_{0 j}$ является точкой 0 , а интегрирование идет от $-\pi$ до $\pi$ по $\varphi$ ) в первом слагаемом и в силу симметрии точек $\varphi_{0 i}$ относительно точки $\varphi_{0 j}$ (см. рис. 2) во втором слагаемом.

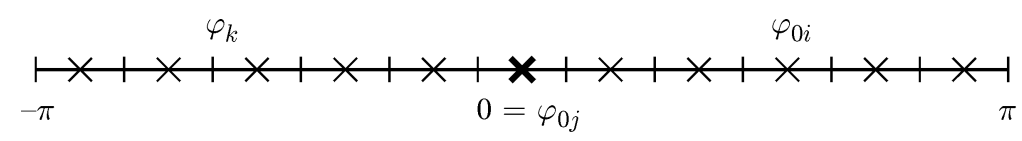

Рис. 2

Для $\eta_{2}\left(M_{0 j, 0 m}\right)$ с учетом неравенств $(2.52)$ получаем

$$
\begin{aligned}
\eta_{2}\left(M_{0 j, 0 m}\right) & \leqslant \sum_{i=1}^{2 n} \sum_{k=1}^{n} \int_{I_{i, k}} \frac{\left|\varphi-\varphi_{0 i}\right|}{\widetilde{r}_{M M_{0 j, 0 m}}^{2}} d \theta d \varphi \\
& =\int_{I_{j, m}} \frac{\left|\varphi-\varphi_{0 i}\right|}{\widetilde{r}_{M M_{0 j, 0 m}}^{2}} d \theta d \varphi+\sum_{\substack{i=1 \\
(i, k) \neq(j, m)}}^{2 n} \sum_{\substack{k=1 \\
I_{i, k}}} \frac{\left|\varphi-\varphi_{0 i}\right|}{\widetilde{r}_{M M_{0 j, 0 m}}^{2}} d \theta d \varphi
\end{aligned}
$$

Переходя опять к полярньм координатам (2.38), видим, что

$$
\eta_{2}\left(M_{0 j, 0 m}\right) \leqslant B_{2} \frac{h \ln n}{\sin ^{2} \theta_{0 m}}
$$

Для оценки $\eta_{1}\left(M_{0 j, 0 m}\right)$ запишем его в следуюшем виде:

$$
\begin{aligned}
\eta_{1}\left(M_{0 j, 0 m}\right) \leqslant & \left|\sum_{i=1}^{2 n} \sum_{k=1}^{n} \int_{I_{i, k}} \frac{\left[g_{\varphi}^{\prime}(M)-g_{\varphi}^{\prime}\left(M_{0 i, 0 k}\right)\right]\left(\varphi-\varphi_{0 i}\right)}{\widetilde{r}_{M M_{0 j, 0 m}}^{3}}\left(\sin \theta-\sin \theta_{0 m}\right) d \theta d \varphi\right| \\
& +\left|\sin \theta_{0 m}\right|\left|\sum_{i=1}^{2 n} \sum_{k=1}^{n} \int_{I_{i, k}} \frac{\left[g_{\varphi}^{\prime}(M)-g_{\varphi}^{\prime}\left(M_{0 i, 0 k}\right)\right]\left(\varphi-\varphi_{0 i}\right)}{\widetilde{r}_{M M_{0 j, 0 m}}^{3}} d \theta d \varphi\right|_{(2.64)}
\end{aligned}
$$


Опять используя неравенства $(2.51)$ и выделяя слагаемое $(j, m)$, получим оценку

$$
\eta_{1}\left(M_{0 j, 0 m}\right) \leqslant B_{3} \frac{h^{\alpha}}{\sin ^{2} \theta_{0 m}}
$$

Таким образом, для $I_{1,1}^{* *}\left(M_{0 j, 0 m}\right)$ в формуле (2.57) получена оценка такого же типа, как и оценка $(2.59)$ для слагаемого $I_{1,1}^{*}\left(M_{0 j, 0 m}\right)$ в этой формуле.

Наконец, для исследования $I_{1,1}^{* * *}\left(M_{0 j, 0 m}\right)$, сделав для него такие же преобразования, как и для $I_{1,1}^{* *}\left(M_{0 j, 0 m}\right)$, придем к рассмотрению величин, аналогичных величинам $\eta_{p}\left(M_{0 j, 0 m}\right), p=1,2,3$, с заменой $\left(\varphi-\varphi_{0 i}\right)$ на $\left(\theta-\theta_{0 k}\right)$. Для первых двух слагаемых получим такие же оценки, а для третьего слагаемого, предполагая $\theta_{0 m}<\pi / 2$, получим (см. рис. 3 )

$$
\begin{aligned}
& \left|\sum_{i=1}^{2 n} \sum_{k=1}^{n} \int_{I_{i, k}} \frac{\theta-\theta_{0 k}}{\widetilde{r}_{M M_{0 j, 0 m}}^{3}} d \theta d \varphi\right|=\left|\sum_{i=1}^{2 n} \sum_{k=2 m}^{n} \int_{I_{i, k}} \frac{\theta-\theta_{0 k}}{\widetilde{r}_{M M_{0 j, 0 m}}^{3}} d \theta d \varphi\right| \\
& \leqslant \frac{h}{\sin ^{3} \theta_{0 m}} \int_{\alpha_{1}}^{\alpha_{2}} d \alpha \int_{\left(-\theta_{0 m}+\theta_{2 m}\right) /|\sin \alpha|} \frac{d r}{r^{2}} \\
& \leqslant B_{4} \frac{h}{\sin ^{3} \theta_{0 m} \rho^{*}\left(\theta_{0 m}\right)} \text {. } \\
& \theta_{02 m-1}
\end{aligned}
$$

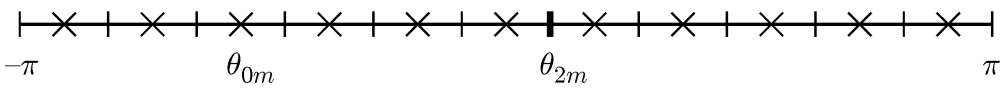

Рис. 3

С учетом множителя $\sin \theta_{0 m}$ окончательно получаем

$$
I_{1,1}^{* * *}\left(M_{0 j, 0 m}\right) \leqslant B_{5} \frac{h}{\sin ^{3} \theta_{0 m} \rho^{*}\left(\theta_{0 m}\right)} .
$$

Из всех приведенных выкладок следует справедливость теоремы.

ЗАмЕчАниЕ 2.1. Численный эксперимент, проведенный для сферы в случаях, когда известны спектральные соотношения для ядра $K_{N}\left(M, M_{0}\right)$, показьвает справедливость теоремы 2.3 и, более того, показывает справедливость результата

$$
\sum_{j=1}^{2 n} \sum_{m=1}^{n}\left|I_{N}\left(M_{0 j, 0 m}\right)-S_{N, n}\left(M_{0 j, 0 m}\right)\right| s_{\sigma_{j, m}} \rightarrow 0 \text { при } n \rightarrow \infty
$$

которьй, к сожалению, не следует из оценок, полученных в процессе доказательства теоремы 2.3. Доказательству такого результата препятствует оценка (2.67). 
Вычислительный эксперимент проводился с использованием соотношений [19]:

$$
\begin{gathered}
\frac{1}{4 \pi} \int_{C_{R}^{2}} K_{N}\left(M, M_{0}\right) Y_{n}(\theta, \varphi) d \sigma_{M}=-\frac{n(n+1)}{R(2 n+1)} Y_{n}\left(\theta_{0}, \varphi_{0}\right), \quad n=0,1,2, \ldots \\
Y_{n}(\theta, \varphi)=\sum_{m=0}^{n}\left(A_{m, n} \cos (m \varphi)+B_{m, n} \sin (m \varphi)\right) P_{n}^{m}(\cos \theta) \\
P_{n}^{m}(\mu)=\left(1-\mu^{2}\right)^{m / 2} P_{n}^{(m)}(\mu), \quad m=0, \ldots, n, \quad n=0,1, \ldots \\
P_{n}(\mu)=\frac{1}{2^{n} n !} \frac{d^{n}}{d \mu^{n}}\left(\mu^{2}-1\right), \quad n=0,1, \ldots
\end{gathered}
$$

где $Y_{n}(\theta, \varphi)$ - сферическая функция порядка $n ; A_{m, n}, B_{m, n}$ - произвольные числа; $P_{n}^{m}(\mu)$ - присоединенные функции Лежандра; $P_{n}(\mu)$ - полиномы Лежандра степени $n$.

Например, рассматривалась функция $Y_{1}(\theta, \varphi)$ при $A_{0,1}=A_{1,1}=B_{0,1}=$ $B_{1,1}=1$. Затем интеграл в левой части заменялся квадратурной суммой

$$
S_{N, n}\left(M_{0 j, 0 m}\right)=S_{N, n}\left(\theta_{0 m}, \varphi_{0 j}\right)
$$

(см. (2.15)) при $g\left(M_{0 i, 0 k}\right)=Y_{1}\left(\theta_{0 k}, \varphi_{0 i}\right)$. Полученные результаты вычислений даны в табл. 1 , где в столбце 1 указано разбиение по $\theta \in[0, \pi]$, в столбце 2 - по $\varphi \in[0,2 \pi]$, в столбце 3 указаны значения сумм

$$
\sum_{j=1}^{2 n} \sum_{m=1}^{n}\left|\frac{1}{4 \pi} S_{N, n}\left(\theta_{0 m}, \varphi_{0 j}\right)\right| \Delta \sigma_{j, m}
$$

в столбце 4 - значения сумм

$$
\sum_{j=1}^{2 n} \sum_{m=1}^{n}\left|\frac{2}{3} Y_{1}\left(\theta_{0 m}, \varphi_{0 j}\right)\right| \Delta \sigma_{j, m}
$$

в столбце 5 - значения сумм

$$
\sum_{j=1}^{2 n} \sum_{m=1}^{n}\left|\frac{1}{4 \pi} S_{N, n}\left(\theta_{0 m}, \varphi_{0 j}\right)+\frac{2}{3} Y_{1}\left(\theta_{0 m}, \varphi_{0 j}\right)\right| \Delta \sigma_{j, m}
$$

а в столбце 6 указаны величины

$$
\max _{(j, m)}\left|\frac{1}{4 \pi} S_{N, n}\left(\theta_{0 m}, \varphi_{0 j}\right)+\frac{2}{3} Y_{1}\left(\theta_{0 m}, \varphi_{0 j}\right)\right|
$$

где $\Delta \sigma_{j, m}-$ площадь поверхности $\sigma_{j, m}$. 
ТАБЛИЦА 1

\begin{tabular}{|c|c|c|c|c|c|}
\hline 1 & 2 & 3 & 4 & 5 & 6 \\
\hline 10 & 20 & 90.40412 & 91.81063 & 2.034871 & 1.943787 \\
\hline 15 & 30 & 90.70240 & 91.40501 & 1.209499 & 2.093547 \\
\hline 20 & 40 & 90.89680 & 91.33085 & 0.862051 & 2.154906 \\
\hline 25 & 50 & 90.94277 & 91.24721 & 0.669597 & 2.195151 \\
\hline 30 & 60 & 91.00057 & 91.23430 & 0.547807 & 2.217790 \\
\hline 35 & 70 & 91.02794 & 91.21292 & 0.464127 & 2.231807 \\
\hline 40 & 80 & 91.04869 & 91.20231 & 0.403166 & 2.241108 \\
\hline 45 & 90 & 91.06531 & 91.19571 & 0.356936 & 2.250282 \\
\hline 50 & 100 & 91.07541 & 91.18868 & 0.320761 & 2.256694 \\
\hline 55 & 110 & 91.08763 & 91.18690 & 0.291534 & 2.261319 \\
\hline 60 & 120 & 91.09575 & 91.18363 & 0.267480 & 2.264742 \\
\hline 65 & 130 & 91.10316 & 91.18303 & 0.247167 & 2.268629 \\
\hline 70 & 140 & 91.10955 & 91.18195 & 0.229727 & 2.271567 \\
\hline 75 & 150 & 91.11315 & 91.17981 & 0.214569 & 2.273816 \\
\hline
\end{tabular}

Далее, рассмотрим численное решение уравнения

$$
\frac{1}{4 \pi} \int_{C_{R}^{2}} K_{N}\left(M, M_{0}\right) g(M) d \sigma_{M}=-\frac{2}{3} Y_{1}\left(\theta_{0}, \varphi_{0}\right), \quad \theta_{0} \in[0, \pi], \varphi_{0} \in[0,2 \pi]
$$

для которого точное решение $g(M)=Y_{1}(\theta, \varphi)$. Численное решение этого уравнения получим из системы уравнений

$$
\begin{gathered}
\gamma_{0 n}+\frac{1}{4 \pi} \sum_{i=1}^{2 n} \sum_{k=1}^{n} g_{n}\left(\theta_{0 k}, \varphi_{0 i}\right) \int_{\sigma_{i, k}} K_{N}\left(M, M_{0 j, 0 m}\right) d \sigma_{M}=-\frac{2}{3} Y_{1}\left(\theta_{0 m}, \varphi_{0 j}\right) \\
m=1, \ldots, n, \quad j=1, \ldots, 2 n \\
\sum_{i=1}^{2 n} \sum_{k=1}^{n} g_{n}\left(\theta_{0 k}, \varphi_{0 i}\right) \Delta \sigma_{i, k}=0
\end{gathered}
$$

Результаты численных расчетов даны в табл. 2, где столбцы 1 и 2 те же, что в табл. 1, в столбце 3 указаны значения сумм

$$
\sum_{i=1}^{2 n} \sum_{k=1}^{n}\left|g_{n}\left(\theta_{0 k}, \varphi_{0 i}\right)\right| \Delta \sigma_{i, k}
$$

в столбце 4 - значения сумм

$$
\sum_{i=1}^{2 n} \sum_{k=1}^{n}\left|Y_{1}\left(\theta_{0 k}, \varphi_{0 i}\right)\right| \Delta \sigma_{i, k}
$$

в столбце 5 - значения сумм

$$
\sum_{i=1}^{2 n} \sum_{k=1}^{n}\left|g_{n}\left(\theta_{0 k}, \varphi_{0 i}\right)-Y_{1}\left(\theta_{0 k}, \varphi_{0 i}\right)\right| \Delta \sigma_{i, k}
$$


в столбце 6 - значения величины

$$
\max _{(i, k)}\left|g_{n}\left(\theta_{0 k}, \varphi_{0 i}\right)-Y_{1}\left(\theta_{0 k}, \varphi_{0 i}\right)\right|
$$

\section{ТАБЛИЦА 2}

\begin{tabular}{|c|c|c|c|c|c|}
\hline 1 & 2 & 3 & 4 & 5 & 6 \\
\hline 10 & 20 & 11.1044 & 10.9591 & 0.1522 & 0.0570 \\
\hline 15 & 30 & 10.9765 & 10.9107 & 0.0722 & 0.0404 \\
\hline 20 & 40 & 10.9398 & 10.9018 & 0.0440 & 0.0311 \\
\hline 25 & 50 & 10.9171 & 10.8918 & 0.0307 & 0.0253 \\
\hline 30 & 60 & 10.9087 & 10.8903 & 0.0234 & 0.0213 \\
\hline 35 & 70 & 10.9017 & 10.8877 & 0.0188 & 0.0184 \\
\hline 40 & 80 & 10.8977 & 10.8865 & 0.0156 & 0.0162 \\
\hline 45 & 90 & 10.8949 & 10.8857 & 0.0134 & 0.0144 \\
\hline
\end{tabular}

В следующей теореме установлено представляющее интерес в различных теоретических и вычислительных задачах спектральное соотношение.

ТЕОРЕМА 2.4. На единичной сфере справедливо следующее спектральное соотношение:

$$
\frac{1}{4 \pi} \int_{C_{R=1}^{2}} \frac{1}{r_{M M_{0}}^{3}} Y_{n}(\theta, \varphi) d \sigma_{M}=-\frac{2 n+1}{4} Y_{n}\left(\theta_{0}, \varphi_{0}\right), \quad n=0,1, \ldots
$$

ДокАЗАТЕЛЬСтво. Известно [19; гл. 5, §2], [25; гл. II, §2, п. 112] следующее спектральное соотношение:

$$
\frac{1}{4 \pi} \int_{C_{R=1}^{2}} \frac{1}{r_{M M_{0}}} Y_{n}(\theta, \varphi) d \sigma_{M}=\frac{1}{2 n+1} Y_{n}\left(\theta_{0}, \varphi_{0}\right), \quad n=0,1, \ldots
$$

Теперь, используя соотношения (2.69), (2.76) и представление интеграла в (2.69) в виде (2.30) для $R=1$, получим

$$
\begin{aligned}
\frac{1}{4 \pi} & \int_{C_{R=1}^{2}} \frac{1}{r_{M M_{0}}^{3}} Y_{n}(\theta, \varphi) d \sigma_{M} \\
& =-\frac{(n+1) n}{2 n+1} Y_{n}\left(\theta_{0}, \varphi_{0}\right)-\frac{1}{4(2 n+1)} Y_{n}\left(\theta_{0}, \varphi_{0}\right) \\
& =-\frac{(2 n+1)^{2}}{4(2 n+1)} Y_{n}\left(\theta_{0}, \varphi_{0}\right)=-\frac{2 n+1}{4} Y_{n}\left(\theta_{0}, \varphi_{0}\right), \quad n=0,1, \ldots
\end{aligned}
$$

Теорема доказана. 


\section{§3. Обобщение понятия сингулярного интеграла}

В процессе доказательства теоремы 2.3 была рассмотрена следующая конструкция. Дан двумерный интеграл вида

$$
I\left(M_{0}\right)=\int_{D} \frac{f\left(M_{0}, \varphi\right)}{r_{M M_{0}}^{2}} g(M) d \sigma_{M},
$$

где $D$ - область в плоскости $O x y ; \varphi$ - полярный угол точки $M$ относительно точки $M_{0}, f\left(M_{0}, \varphi\right)$ - функция, ограниченная на $D \times D$ как функция точек $M$ и $M_{0}$ из области $D$, непрерывная в $D \backslash M_{0}$ и являющаяся достаточно гладкой функцией в полярных координатах с центром в точке $M_{0}$ на плоскости $O x y$; функция $g(M) \in H(\alpha)$ - плотность интеграла $I\left(M_{0}\right)$ и $d \sigma_{M}=d x d y$. Пусть $D$ является прямоугольником и интеграл $I\left(M_{0}\right)$ понимается в некотором смысле, обобшающем понятие несобственного двумерного интеграла. Зададим на $D$ каноническое разбиение на прямоугольники $D_{i, k}, i=1, \ldots, n_{1}, k=1, \ldots, n_{2}$, с центрами в точ$\operatorname{\kappa ax} M_{0 i, 0 k}$ и со сторонами $h_{1}$ и $h_{2}$ такими, что $0<q_{1} \leqslant h_{1} / h_{2}, h_{2} / h_{1} \leqslant q_{2}<+\infty$, где $q_{1}$ и $q_{2}$ - некоторые данные числа, и пусть $h=\min \left(h_{1} ; h_{2}\right)$.

Возьмем теперь для интеграла $I\left(M_{0}\right)$ квадратурную сумму

$$
\begin{gathered}
S_{h}\left(M_{0 j, 0 m}\right)=\sum_{i=1}^{n_{1}} \sum_{k=1}^{n_{2}} g\left(M_{0 i, 0 k}\right) \int_{D_{i, k}} \frac{f\left(M_{0 j, 0 m}, \varphi_{0 j, 0 m}\right)}{r_{M M_{0 j, 0 m}}^{2}} d \sigma_{M} \\
j=1, \ldots, n_{1}, \quad m=1, \ldots, n_{2}
\end{gathered}
$$

где $\varphi_{0 j, 0 m}$ - полярный угол точки $M$ интегрирования относительно точки $M_{0 j, 0 m}$. Справедлива следующая

ТЕОРема 3.1. Пусть функция $g(M)$ принадлежсит классу $H(\alpha)$ на $D, 0<$ $\alpha \leqslant 1$. Тогда справедливо неравенство

$$
\left|I\left(M_{0 j, 0 m}\right)-S_{h}\left(M_{0 j, 0 m}\right)\right| \leqslant C h^{\alpha}|\ln h|
$$

для всех точек $M_{0 j, 0 m}$.

ДокАЗАТЕЛЬСТво. Используя формулы (3.1) и (3.2), можно записать

$$
\begin{aligned}
& \left|I\left(M_{0 j, 0 m}\right)-S_{h}\left(M_{0 j, 0 m}\right)\right| \\
& \quad=\left|\sum_{i=1}^{n_{1}} \sum_{k=1}^{n_{2}} \int_{D_{i, k}} \frac{f\left(M_{0 j, 0 m}, \varphi_{0 j, 0 m}\right)\left[g(M)-g\left(M_{0 i, 0 k}\right)\right]}{r_{M M_{0 j, 0 m}}^{2}} d x d y\right| \\
& \quad \leqslant B A \int_{D_{j, m}} \frac{d x d y}{r_{M M_{0 j, 0 m}}^{2-\alpha}}+B A A_{1}^{\alpha} \frac{h^{\alpha}}{(\sqrt{2})^{\alpha}} \sum_{\substack{i=1 \\
(i, k) \neq(j, m)}}^{n_{1}} \sum_{\substack{k=1 \\
n_{2}}} \int_{D_{i, k}} \frac{d x d y}{r_{M M_{0 j, 0 m}}^{2}}=I_{1}+I_{2},
\end{aligned}
$$

где $B=\sup _{M_{0}, M \in D}\left|f\left(M_{0}, \varphi\right)\right|, A$ - константа в условии Гёльдера для функции $g(M)$, а $A_{1}>0$ - такое число, что $h_{1}, h_{2} \leqslant A_{1} h$ при $n_{1}, n_{2} \rightarrow \infty$. 
Для оценки слагаемых $I_{1}$ и $I_{2}$ в формуле (3.4) введем на плоскости $O x y$ полярные координаты с центром в точке $M_{0 j, 0 m}$. Получим

$$
I_{1} \leqslant C_{1} \int_{0}^{2 \pi} d \varphi \int_{0}^{h} \frac{d r}{r^{1-\alpha}}=C_{1} 2 \pi h^{\alpha}
$$

где $C_{1}=B A$.

Далее,

$$
I_{2} \leqslant C_{2} h^{\alpha} \int_{0}^{2 \pi} d \varphi \int_{h / 2}^{R} \frac{d r}{r}=C_{2} 2 \pi h^{\alpha}\left(\ln R-\ln \frac{h}{2}\right) \leqslant C_{3} h^{\alpha}|\ln h|,
$$

где $C_{2}=C_{1}\left(A_{1} / \sqrt{2}\right)^{\alpha}, C_{3}=4 \pi C_{2}$, а $R$ - радиус круга с центром в точке $M_{0 j, 0 m}$, содержашем область $D$. Теорема доказана.

Теперь посмотрим, в каком едином смысле можно понимать интегралы вида (3.1). Для этого рассмотрим конкретные примеры.

Рассмотрим вначале двумерньй интеграл

$$
I\left(M_{0}\right)=\int_{D} \frac{1}{r_{M M_{0}}^{2}} \frac{x-x_{0}}{r_{M M_{0}}} d x d y
$$

где $D$ - некоторая ограниченная односвязная область, $M_{0} \in D$.

Видно, что подьнтегральная функция в интеграле (3.7) неограничена в точке $M_{0}$ и в обычном смысле он не существует. По аналогии с одномерным сингулярньм интегралом было предложено поступить следующим образом: вырезать $\varepsilon$-окрестность $O\left(M_{0}, \varepsilon\right)$ точки $M_{0}$ и взять интеграл (3.7) по оставшемуся множеству $D \backslash O\left(M_{0}, \varepsilon\right)$; затем устремить $\varepsilon$ к нулю и если предел существует, то его и обозначить $I\left(M_{0}\right)$, т.е. определить этот интеграл по формуле

$$
I\left(M_{0}\right)=\lim _{\varepsilon \rightarrow 0} \int_{D \backslash O\left(M_{0}, \varepsilon\right)} \frac{1}{r_{M M_{0}}^{2}} \frac{x-x_{0}}{r_{M M_{0}}} d x d y
$$

где $M_{0} \in D$.

Переходя к полярным координатам с центром в точке $M_{0}$ на плоскости $O x y$, получим

$$
\begin{aligned}
& \lim _{\varepsilon \rightarrow 0} \int_{D \backslash O\left(M_{0}, \varepsilon\right)} \frac{1}{r_{M M_{0}}^{2}} \frac{x-x_{0}}{r_{M M_{0}}} d x d y=\lim _{\varepsilon \rightarrow 0} \int_{0}^{2 \pi} d \varphi \int_{\varepsilon}^{\rho\left(M_{0}, \varphi\right)} \frac{1}{r^{2}} \cos \varphi r d r \\
& \quad= \lim _{\varepsilon \rightarrow 0} \int_{0}^{2 \pi} \cos \varphi\left(\ln \rho\left(M_{0}, \varphi\right)-\ln \varepsilon\right) d \varphi=\lim _{\varepsilon \rightarrow 0} \int_{0}^{2 \pi} \cos \varphi \ln \rho\left(M_{0}, \varphi\right) d \varphi \\
& \quad=\int_{0}^{2 \pi} \cos \varphi \ln \rho\left(M_{0}, \varphi\right) d \varphi
\end{aligned}
$$

так как

$$
\int_{0}^{2 \pi} \cos \varphi d \varphi=0
$$


а функция $r=\rho\left(M_{0}, \varphi\right)$ описывает границу области $D$ в выбранной системе координат.

Таким образом, интеграл $I\left(M_{0}\right)$, понимаемый в смысле равенства $(3.8)$, имеет значение

$$
I\left(M_{0}\right)=\int_{0}^{2 \pi} \cos \varphi \ln \rho\left(M_{0}, \varphi\right) d \varphi
$$

Аналогично можно определить интеграл вида

$$
I\left(M_{0}\right)=\int_{D} \frac{1}{r_{M M_{0}}^{2}} f\left(M_{0}, \varphi\right) d x d y
$$

где $\varphi$ - полярный угол точки $M$ по отношению к точке $M_{0}$, а функция $f\left(M_{0}, \varphi\right)$ такая, как указано в (3.1). Как известно [22; 5$]$, интеграл (3.12) в смысле (3.8) существует тогда и только тогда, когда выполняется равенство

$$
\int_{0}^{2 \pi} f\left(M_{0}, \varphi\right) d \varphi=0
$$

где $M_{0} \in D$. Если же равенство (3.13) не выполняется для некоторой точки $M_{0} \in D$, то говорят $[22 ; \S 5]$, что интеграл $(3.12)$ не существует. С другой стороны, как было показано в теореме 3.1 , квадратурные формулы (исследованные там) сходятся к точному значению интеграла $I\left(M_{0}\right)$, не обязательно понимаемому в смысле равенства (3.13). Поэтому имеет смысл ввести следующее определение.

ОПРЕДЕЛЕНИЕ 3.1. Пусть характеристика $f\left(M_{0}, \varphi\right)$ такая, как это указано после (3.1). Будем полагать

$$
I\left(M_{0}\right)=\lim _{\varepsilon \rightarrow 0}\left[\int_{D \backslash O\left(M_{0}, \varepsilon\right)} \frac{f\left(M_{0}, \varphi\right)}{r_{M M_{0}}^{2}} d x d y+\ln \varepsilon \int_{0}^{2 \pi} f\left(M_{0}, \varphi\right) d \varphi\right]
$$

если предел существует, и по-прежнему будем обозначать его в виде (3.12).

Сразу отметим, что если равенство (3.13) выполняется, то интеграл $I\left(M_{0}\right)$, понимаемый в смысле (3.14), является двумерньм сингулярным интегралом в обычном смысле $[22 ; \S 5]$.

Справедлива следующая

ТЕОРема 3.2. Если характеристика в интеграле (3.1) удовлетворяет сформулированным после (3.1) условиям, то интеграл $I\left(M_{0}\right)$, понимаемый в смысле равенства (3.14), существует.

ДоКАЗАТЕЛЬСТво. Перейдя в (3.14) к полярным координатам с центром в точке $M_{0}$, получим

$$
\begin{aligned}
I\left(M_{0}\right) & =\lim _{\varepsilon \rightarrow 0}\left[\int_{0}^{2 \pi} f\left(M_{0}, \varphi\right) d \varphi \int_{\varepsilon}^{\rho\left(M_{0}, \varphi\right)} \frac{d r}{r}+\ln \varepsilon \int_{0}^{2 \pi} f\left(M_{0}, \varphi\right) d \varphi\right] \\
& =\int_{0}^{2 \pi} f\left(M_{0}, \varphi\right) \ln \rho\left(M_{0}, \varphi\right) d \varphi
\end{aligned}
$$


где $r=\rho\left(M_{0}, \varphi\right)$ - уравнение границы области $D$ в выбранных полярных координатах. Теорема доказана.

В качестве примера рассмотрим интеграл

$$
I^{*}\left(M_{0}\right)=\int_{D} \frac{\sin (\varphi / 2)}{r_{M M_{0}}^{2}} d x d y .
$$

Для характеристики этого интеграла выполняется равенство

$$
\int_{0}^{2 \pi} \sin \frac{\varphi}{2} d \varphi=-\left.2 \cos \frac{\varphi}{2}\right|_{0} ^{2 \pi}=4
$$

Следовательно, интеграл $I^{*}\left(M_{0}\right)$ не существует в обычном смысле [22; 5 ] сингулярного двумерного интеграла, т.е. в смысле равенства (3.8), но существует в смысле равенства (3.14).

Теперь рассмотрим сингулярный интеграл вида

$$
I\left(M_{0}\right)=\int_{D} \frac{f\left(M_{0}, \varphi\right)}{r_{M M_{0}}^{2}} g(M) d x d y,
$$

где функция $g(M)$ называется плотностью этого интеграла, а функция $f\left(M_{0}, \varphi\right)$ - характеристикой.

ОПРЕДЕЛЕНИЕ 3.2. Интеграл (3.18) будем назьвать сингулярным интегралом и понимать его в следующем смысле

$$
I\left(M_{0}\right)=\lim _{\varepsilon \rightarrow 0}\left[\int_{D \backslash O\left(M_{0}, \varepsilon\right)} \frac{f\left(M_{0}, \varphi\right)}{r_{M M_{0}}^{2}} g(M) d x d y+g\left(M_{0}\right) \ln \varepsilon \int_{0}^{2 \pi} f\left(M_{0}, \varphi\right) d \varphi\right] .
$$

Опять можно заметить, что если выполняется равенство (3.13), то интеграл (3.19) будет сингулярным интегралом в обычном смысле [22; 5 5].

Теперь докажем теорему.

ТЕОРема 3.3. Пусть функиия $g(M)$ принадлежсит классу $H(\alpha), 0<\alpha \leqslant 1$, на области $D$, а характеристика $f\left(M_{0}, \varphi\right)$ удовлетворяет условиям, указанным после (3.1). Тогда интеграл (3.19) существует для любой точки $M_{0}$ из области D.

ДокАЗАТЕльство. Преобразуем формулу (3.19) следующим образом:

$$
\begin{aligned}
I\left(M_{0}\right)= & \lim _{\varepsilon \rightarrow 0}\left(\int_{D \backslash O\left(M_{0}, \varepsilon\right)} \frac{f\left(M_{0}, \varphi\right)}{r_{M M_{0}}^{2}}\left[g(M)-g\left(M_{0}\right)\right] d x d y\right) \\
& +\lim _{\varepsilon \rightarrow 0}\left(g\left(M_{0}\right) \int_{D \backslash O\left(M_{0}, \varepsilon\right)} \frac{f\left(M_{0}, \varphi\right)}{r_{M M_{0}}^{2}} d x d y+g\left(M_{0}\right) \ln \varepsilon \int_{0}^{2 \pi} f\left(M_{0}, \varphi\right) d \varphi\right) \\
= & I_{1}\left(M_{0}\right)+I_{2}\left(M_{0}\right) .
\end{aligned}
$$

В формуле (3.20) для интеграла $I_{2}\left(M_{0}\right)$ с использованием (3.15) получаем

$$
I_{2}\left(M_{0}\right)=g\left(M_{0}\right) \int_{0}^{2 \pi} f\left(M_{0}, \varphi\right) \ln \rho\left(M_{0}, \varphi\right) d \varphi .
$$

Интеграл $I_{1}\left(M_{0}\right)$ является абсолютно сходящимся, так как $g(M) \in H(\alpha)$ на $D$. Теорема доказана. 
ЗАмЕчАниЕ 3.1. Теорема 3.1 справедлива для интеграла (3.1), понимаемого в смысле (3.19).

ЗАмечАниЕ 3.2. Интеграл (3.1), понимаемый в смысле (3.19), обладает свойством линейности на множестве функций из $H(\alpha)$ на $D$, т.е. если $g(M)$ и $\eta(M)$ принадлежат $H(\alpha)$ на $D$, то

$$
\begin{aligned}
\int_{D} & \frac{f\left(M_{0}, \varphi\right)}{r_{M M_{0}}^{2}}[A g(M)+B \eta(M)] d x d y \\
= & A \int_{D} \frac{f\left(M_{0}, \varphi\right)}{r_{M M_{0}}^{2}} g(M) d x d y+B \int_{D} \frac{f\left(M_{0}, \varphi\right)}{r_{M M_{0}}^{2}} \eta(M) d x d y .
\end{aligned}
$$

Рассмотрим теперь с аналогичной точки зрения интеграл (2.11). Вначале рассмотрим интеграл вида

$$
I^{*}\left(M_{0}\right)=\int_{D} \frac{f\left(M_{0}, \varphi\right)}{r_{M M_{0}}^{3}} d x d y
$$

где функция $f\left(M_{0}, \varphi\right)$, называемая характеристикой интеграла, такая, как указано после $(3.1)$, в частности, она может быть константой. Определим $I^{*}\left(M_{0}\right)$ следуюшим образом:

$$
I^{*}\left(M_{0}\right)=\lim _{\varepsilon \rightarrow 0}\left[\int_{D \backslash O\left(M_{0}, \varepsilon\right)} \frac{f\left(M_{0}, \varphi\right)}{r_{M M_{0}}^{3}} d x d y-\frac{1}{\varepsilon} \int_{0}^{2 \pi} f\left(M_{0}, \varphi\right) d \varphi\right] .
$$

Как и для интеграла (2.9), показывается, что этот интеграл сушествует.

Теперь рассмотрим интеграл

$$
I^{*}\left(M_{0}\right)=\int_{D} \frac{f\left(M_{0}, \varphi\right)}{r_{M M_{0}}^{3}} g(M) d x d y, \quad M_{0} \in \sigma
$$

обобщающий интеграл (2.11). Пусть $g(M) \in H_{1}(\alpha)$ на $D$, и рассмотрим по аналогии с формулой (2.12) предел

$$
\begin{aligned}
\lim _{\varepsilon \rightarrow 0}\left[\int_{D \backslash O\left(M_{0}, \varepsilon\right)} \frac{f\left(M_{0}, \varphi\right)}{r_{M M_{0}}^{3}} g(M) d x d y-\frac{g\left(M_{0}\right)}{\varepsilon} \int_{0}^{2 \pi} f\left(M_{0}, \varphi\right) d \varphi\right] \\
=\lim _{\varepsilon \rightarrow 0}\left[\int_{D \backslash O\left(M_{0}, \varepsilon\right)} \frac{f\left(M_{0}, \varphi\right)}{r_{M M_{0}}^{3}} \Delta\left(M, M_{0}\right) d x d y\right] \\
\quad+\lim _{\varepsilon \rightarrow 0}\left[g\left(M_{0}\right)\left(\int_{D \backslash O\left(M_{0}, \varepsilon\right)} \frac{f\left(M_{0}, \varphi\right)}{r_{M M_{0}}^{3}} d x d y-\frac{1}{\varepsilon} \int_{0}^{2 \pi} f\left(M_{0}, \varphi\right) d \varphi\right)\right] \\
\quad+\lim _{\varepsilon \rightarrow 0}\left[g_{x}^{\prime}\left(M_{0}\right) \int_{D \backslash O\left(M_{0}, \varepsilon\right)} \frac{f\left(M_{0}, \varphi\right) \cos \varphi}{r_{M M_{0}}^{2}} d x d y\right] \\
\quad+\lim _{\varepsilon \rightarrow 0}\left[g_{y}^{\prime}\left(M_{0}\right) \int_{D \backslash O\left(M_{0}, \varepsilon\right)} \frac{f\left(M_{0}, \varphi\right) \sin \varphi}{r_{M M_{0}}^{2}} d x d y\right],
\end{aligned}
$$

где $\Delta\left(M, M_{0}\right)=g(M)-g\left(M_{0}\right)-g_{x}^{\prime}\left(M_{0}\right)\left(x-x_{0}\right)-g_{y}^{\prime}\left(M_{0}\right)\left(y-y_{0}\right)$. 
В силу неравенства (2.14) первое слагаемое имеет предел. Второе слагаемое имеет предел, как это указано для формулы (3.23), а вот третье и четвертое слагаемые имели бы предел, если бы выполнялись равенства

$$
\int_{0}^{2 \pi} f\left(M_{0}, \varphi\right) \cos \varphi d \varphi=\int_{0}^{2 \pi} f\left(M_{0}, \varphi\right) \sin \varphi d \varphi=0
$$

Так как равенства (3.26) не обязательно вьполняются, то интеграл (3.24) надо определить следующим образом.

ОПРЕДЕЛЕНИЕ 3.3. Пусть частные производные функции $g(M)$ существуют в каждой точке области $D$. Тогда интеграл (3.24) понимаем следующим образом:

$$
\begin{aligned}
I^{*}\left(M_{0}\right)= & \int_{D} \frac{f\left(M_{0}, \varphi\right)}{r_{M M_{0}}^{3}} g(M) d x d y \\
= & \lim _{\varepsilon \rightarrow 0}\left[\int_{D \backslash O\left(M_{0}, \varepsilon\right)} \frac{f\left(M_{0}, \varphi\right)}{r_{M M_{0}}^{3}} g(M) d x d y\right. \\
& -\frac{g\left(M_{0}\right)}{\varepsilon} \int_{0}^{2 \pi} f\left(M_{0}, \varphi\right) d \varphi+g_{x}^{\prime}\left(M_{0}\right) \ln \varepsilon \int_{0}^{2 \pi} f\left(M_{0}, \varphi\right) \cos \varphi d \varphi \\
& \left.+g_{y}^{\prime}\left(M_{0}\right) \ln \varepsilon \int_{0}^{2 \pi} f\left(M_{0}, \varphi\right) \sin \varphi d \varphi\right] .
\end{aligned}
$$

Принимая во внимание формулу (3.25) и теорему 3.2 , видим теперь, что справедлива следуюшая теорема.

Теорема 3.4. Пусть характеристика $f\left(M_{0}, \varphi\right)$ в интеграле (3.24) такая, как указано после (3.1), а плотность $g(M)$ принадлежсит классу $H_{1}(\alpha)$ на D. Тогда интеграл (3.24), понимаемый в смысле равенства (3.27), существует.

ЗАмЕчАНИЕ 3.3. Определение 3.3 является обобщением определения 2.2, так как если взять $f\left(M_{0}, \varphi\right) \equiv 1$, то формула (3.27) перейдет в формулу (2.12).

ЗАмЕчАниЕ 3.4. Если проанализировать доказательство сходимости квадратурных формул для гиперсингулярного интеграла на сфере, то ясно, что для гиперсингулярного интеграла (3.22) будет справедлив аналогичный результат для некоторого класса характеристик. Чтобы определить этот класс, заметим вначале, что в этом доказательстве сушественную роль играло свойство функций $\cos \varphi$ и $\sin \varphi: \sin (\varphi+\pi)=-\sin \varphi$ и $\cos (\varphi+\pi)=-\cos \varphi$. Поэтому введем следуюшее определение.

ОПРЕДЕЛЕНИЕ 3.4. Назовем функцию $f\left(M_{0}, \varphi\right)$ четной или нечетной относительно $\pi$, если для нее выполняется соответственно равенство

$$
\begin{aligned}
& f\left(M_{0}, \varphi+\pi\right)=f\left(M_{0}, \varphi\right), \\
& f\left(M_{0}, \varphi+\pi\right)=-f\left(M_{0}, \varphi\right) .
\end{aligned}
$$

Видно, что $\cos \varphi$ и $\sin \varphi$ являются нечетньми относительно $\pi$. Поэтому если $f\left(M_{0}, \varphi\right)$ является четной относительно $\pi$, то $f\left(M_{0}, \varphi\right) \cos \varphi$ и $f\left(M_{0}, \varphi\right) \sin \varphi$ являются нечетньми относительно $\pi$.

Таким образом, справедлива следующая теорема. 
ТЕОРема 3.5. Пусть характеристика $f\left(M_{0}, \varphi\right)$ в интеграле (3.24) является четной относительно $\pi, g(M)$ принадлехсит классу $H_{1}(\alpha)$ на $D$ и область $D$ является прямоугольником. Возьмем для $D$ каноническое разбиение на прямоугольники $D_{i, k}, i=1, \ldots, n_{1}, k=1, \ldots, n_{2}$, с чентрами в точках $M_{0 i, 0 k}$ и суммм

$$
S_{h}\left(M_{0 j, 0 m}\right)=\sum_{i=1}^{n_{1}} \sum_{k=1}^{n_{2}} g\left(M_{0 i, 0 k}\right) \int_{D_{i, k}} \frac{f\left(M_{0 j, 0 m}, \varphi_{0 j, 0 m}\right)}{r_{M M_{0 j, 0 m}}^{3}} d x d y,
$$

где $\varphi_{0 j, 0 т}$ - полярный угол точки $M$ относительно точки $M_{0 j, 0 m}$.

Тогда для всех точек $M_{0 j, 0 m}$ таких, что расстояние $\rho\left(M_{0 j, 0 m}\right.$, гр $\left.D\right)$ от этих точек до границы области $D$ не менее $\delta>0$, выполняется соотночение

$$
\left|I\left(M_{0 j, 0 m}\right)-S_{h}\left(M_{0 j, 0 m}\right)\right| \leqslant C_{\delta} h^{\alpha}|\ln h| .
$$

\section{§4. Гиперсингулярный интеграл и уравнение с ним на торе}

Пример сферы показал, что если параметрическое представление поверхности является периодическим по одной из переменных, то по этой переменной удается доказать равномерную по расчетньп точкам $M_{0 j, 0 m}$ сходимость квадратурных формул типа метода дискретных замкнутых вихревых рамок к точным значениям соответствуюшего гиперсингулярного интеграла. Поэтому целесообразно теперь рассмотреть замкнутую поверхность, параметрическое представление которой периодическое по обеим переменным. Примером такой поверхности является тор.

Итак, пусть теперь поверхность $\sigma$ в уравнении (2.6) является тором. Напишем параметрическое уравнение тора в удобной форме. Геометрически ясно, что тор получается врашением окружности вокруг оси, лежащей в плоскости окружности и не пересекающей ее. Поэтому возьмем в плоскости $O x z$ пространства $O x y z$ окружность радиуса $R_{1}>0$ с центром в точке $(a, 0,0)$ оси $O x, 0<R_{1}<a$. Уравнение этой окружности в плоскости $O x z$ будет

$$
\left\{\begin{array}{l}
z=R_{1} \sin \theta, \\
x=a+R_{1} \cos \theta,
\end{array} \quad 0 \leqslant \theta \leqslant 2 \pi .\right.
$$

Теперь напишем уравнение окружности в плоскости $z=R_{1} \sin \theta$ радиуса $R=a+$ $R_{1} \cos \theta$ и с центром в точке $\left(0,0, R_{1} \sin \theta\right)$ (см. рис. 4$)$.

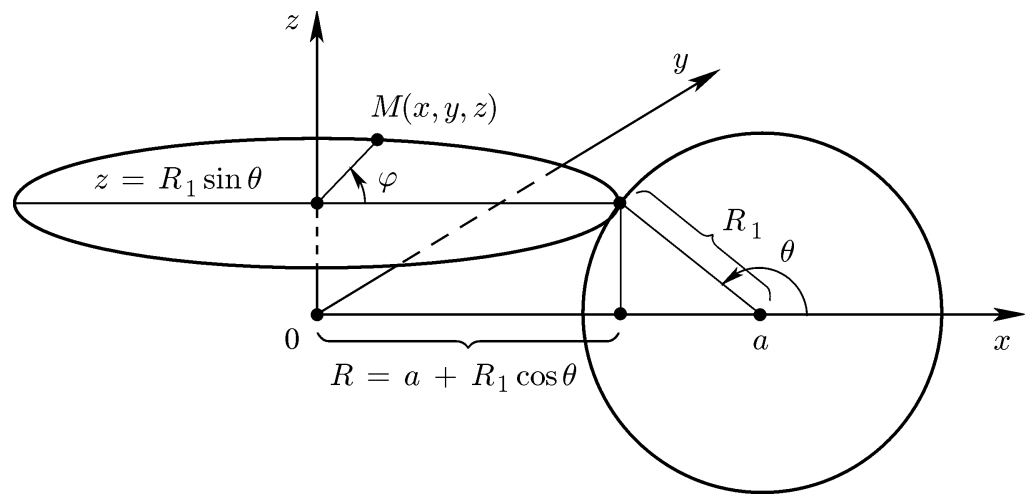

Рис. 4 
Имеем

$$
\left\{\begin{array}{l}
z=R_{1} \sin \theta \\
x=\left(a+R_{1} \cos \theta\right) \cos \varphi \\
y=\left(a+R_{1} \cos \theta\right) \sin \varphi
\end{array}\right.
$$

где $\theta$ - постоянная величина, а $\varphi \in[0,2 \pi]$. Если теперь менять $\theta$ тоже на $[0,2 \pi]$, то система уравнений (4.2) и будет параметрическим представлением тора, где $0 \leqslant \theta, \varphi \leqslant 2 \pi, 0<R_{1}<a$.

Для удобного представления $K_{T, N}\left(M, M_{0}\right)$ на торе, как и в случае сферы, найдем вначале удобное представление для величины $r_{M M_{0}}^{2}$ квадрата расстояния между двумя точками на торе. Имеем

$$
\begin{aligned}
r_{M M_{0}}^{2}= & \left(x-x_{0}\right)^{2}+\left(y_{0}-y\right)^{2}+\left(z_{0}-z\right)^{2} \\
= & {\left[\left(a+R_{1} \cos \theta_{0}\right) \cos \varphi_{0}-\left(a+R_{1} \cos \theta_{0}\right) \cos \varphi\right]^{2} } \\
& +\left[\left(a+R_{1} \cos \theta_{0}\right) \sin \varphi_{0}-\left(a+R_{1} \cos \theta_{0}\right) \sin \varphi\right]^{2}+R_{1}^{2}\left(\sin \theta_{0}-\sin \theta\right)^{2} \\
= & \left(a+R_{1} \cos \theta_{0}\right)^{2}+\left(a+R_{1} \cos \theta\right)^{2} \\
& -2\left(a+R_{1} \cos \theta_{0}\right)\left(a+R_{1} \cos \theta\right)\left(\cos \varphi_{0} \cos \varphi+\sin \varphi_{0} \sin \varphi\right) \\
& +4 R_{1}^{2} \sin ^{2} \frac{\theta_{0}-\theta}{2} \cos ^{2} \frac{\theta_{0}+\theta}{2} \\
= & R_{1}^{2}\left(\cos \theta_{0}-\cos \theta\right)^{2}+4 R_{1}^{2} \sin ^{2} \frac{\theta_{0}-\theta}{2} \cos ^{2} \frac{\theta_{0}+\theta}{2} \\
& +2\left(a+R_{1} \cos \theta_{0}\right)\left(a+R_{1} \cos \theta\right)\left(1-\cos \left(\varphi_{0}-\varphi\right)\right) \\
= & 4 R_{1}^{2} \sin ^{2} \frac{\theta_{0}-\theta}{2} \sin ^{2} \frac{\theta_{0}+\theta}{2}+4 R_{1}^{2} \sin ^{2} \frac{\theta_{0}-\theta}{2} \cos ^{2} \frac{\theta_{0}+\theta}{2} \\
& +4\left(a+R_{1} \cos \theta_{0}\right)\left(a+R_{1} \cos \theta\right) \sin ^{2} \frac{\varphi_{0}-\varphi}{2}
\end{aligned}
$$

Таким образом, окончательно получаем

$$
r_{M M_{0}}^{2}=4\left[R_{1}^{2} \sin ^{2} \frac{\theta_{0}-\theta}{2}+\left(a+R_{1} \cos \theta_{0}\right)\left(a+R_{1} \cos \theta\right) \sin ^{2} \frac{\varphi_{0}-\varphi}{2}\right]
$$

Орт $\bar{n}_{M}$ нормали к тору в точке $M$ найдем как векторное произведение касательных ортов $\bar{\tau}_{\varphi}$ и $\bar{\tau}_{\theta}$ в точке $M$ к соответствующим координатным линиям на торе. Имеем

$$
\left\{\begin{array}{l}
x_{\varphi}^{\prime}=-\left(a+R_{1} \cos \theta\right) \sin \varphi \\
y_{\varphi}^{\prime}=\left(a+R_{1} \cos \theta\right) \cos \varphi \\
z_{\varphi}^{\prime}=0
\end{array}\right.
$$

т.e.

$$
\bar{\tau}_{\varphi}=\frac{x_{\varphi}^{\prime} \bar{i}+y_{\varphi}^{\prime} \bar{j}+z_{\varphi}^{\prime} \bar{k}}{\sqrt{\left(x_{\varphi}^{\prime}\right)^{2}+\left(y_{\varphi}^{\prime}\right)^{2}+\left(z_{\varphi}^{\prime}\right)^{2}}}=-\sin \varphi \bar{i}+\cos \varphi \bar{j}
$$

Аналогично находим

$$
\bar{\tau}_{\theta}=-\sin \theta \cos \varphi \bar{i}-\sin \theta \sin \varphi \bar{j}+\cos \theta \bar{k}
$$


Запишем теперь выражения для внешней нормали в точках $M$ и $M_{0}$ соответственно:

$$
\begin{aligned}
\bar{n}_{M} & =\frac{\bar{\tau}_{\varphi} \times \bar{\tau}_{\theta}}{\left|\bar{\tau}_{\varphi} \times \bar{\tau}_{\theta}\right|}=\cos \theta \cos \varphi \bar{i}+\cos \theta \sin \varphi \bar{j}+\sin \theta \bar{k}, \\
\bar{n}_{M_{0}} & =\frac{\bar{\tau}_{\varphi_{0}} \times \bar{\tau}_{\theta_{0}}}{\left|\bar{\tau}_{\varphi_{0}} \times \bar{\tau}_{\theta_{0}}\right|}=\cos \theta_{0} \cos \varphi_{0} \bar{i}+\cos \theta_{0} \sin \varphi_{0} \bar{j}+\sin \theta_{0} \bar{k} .
\end{aligned}
$$

Далее, имеем

$$
\begin{aligned}
\left(\bar{n}_{M}, \bar{n}_{M_{0}}\right) & =\cos \theta \cos \theta_{0} \cos \left(\varphi_{0}-\varphi\right)+\sin \theta \sin \theta_{0} \\
& =-\cos \theta \cos \theta_{0}\left(1-\cos \left(\varphi_{0}-\varphi\right)\right)+\cos \left(\theta_{0}-\theta\right) \\
& =-2 \sin ^{2} \frac{\varphi_{0}-\varphi}{2} \cos \theta \cos \theta_{0}-\left(1-\cos \left(\theta_{0}-\theta\right)\right)+1 \\
& =1-2 \sin ^{2} \frac{\theta_{0}-\theta}{2}-2 \sin ^{2} \frac{\varphi_{0}-\varphi}{2} \cos \theta \cos \theta_{0} .
\end{aligned}
$$

Так как

$$
\begin{aligned}
\bar{r}_{M M_{0}}= & \left(x_{0}-x\right) \bar{i}+\left(y_{0}-y\right) \bar{j}+\left(z_{0}-z\right) \bar{k} \\
= & {\left[\left(a+R_{1} \cos \theta_{0}\right) \cos \varphi_{0}-\left(a+R_{1} \cos \theta_{0}\right) \cos \varphi\right] \bar{i} } \\
& +\left[\left(a+R_{1} \cos \theta_{0}\right) \sin \varphi_{0}-\left(a-R_{1} \cos \theta_{0}\right) \sin \varphi\right] \bar{j}+R_{1}\left(\sin \theta_{0}-\sin \theta\right) \bar{k}
\end{aligned}
$$

то, принимая во внимание еще формулу (4.8), получим

$$
\begin{array}{rl}
\left(\bar{r}_{M M_{0}}, \bar{n}_{M}\right)=\left[a\left(\cos \varphi_{0}-\cos \varphi\right)+R_{1}\left(\cos \theta_{0} \cos \varphi_{0}-\cos \theta \cos \varphi\right)\right] \cos \theta \cos \varphi \\
+\left[a\left(\sin \varphi_{0}-\sin \varphi\right)+R_{1}\left(\cos \theta_{0} \sin \varphi_{0}-\cos \theta \sin \varphi\right)\right] \cos \theta \sin \varphi \\
+R_{1}\left(\sin \theta_{0} \sin \theta-\sin ^{2} \theta\right) \\
=-2 a \sin \frac{\varphi_{0}-\varphi}{2} \sin \frac{\varphi_{0}+\varphi}{2} \cos \theta \cos \varphi+2 a \sin \frac{\varphi_{0}-\varphi}{2} \cos \frac{\varphi_{0}+\varphi}{2} \cos \theta \sin \varphi \\
& +R_{1}\left[\cos \theta_{0} \cos \varphi_{0} \cos \theta \cos \varphi-\cos ^{2} \theta \cos ^{2} \varphi\right] \\
& +R_{1}\left[\cos \theta_{0} \sin \varphi_{0} \cos \theta \sin \varphi-\cos ^{2} \theta \sin ^{2} \varphi\right]+R_{1}\left(\sin \theta_{0} \sin \theta-\sin ^{2} \theta\right) \\
=2 & 2 a \sin \frac{\varphi_{0}-\varphi}{2} \cos \theta\left(\sin \varphi \cos \frac{\varphi_{0}+\varphi}{2}-\cos \varphi \sin \frac{\varphi_{0}+\varphi}{2}\right) \\
& +R_{1}\left[\cos \theta_{0} \cos \theta\left(\cos \varphi_{0} \cos \varphi+\sin \varphi_{0} \sin \varphi\right)-\cos ^{2} \theta\right] \\
& +R_{1}\left(\sin \theta_{0} \sin \theta-\sin { }^{2} \theta\right) \\
=- & 2 a \sin ^{2} \frac{\varphi_{0}-\varphi}{2} \cos \theta+R_{1} \cos \theta_{0} \cos \theta \cos \left(\varphi_{0}-\varphi\right) \\
& -R_{1}\left(\cos ^{2} \theta+\sin { }^{2} \theta\right)+R_{1} \sin \theta_{0} \sin \theta \\
=- & 2 a \sin ^{2} \frac{\varphi_{0}-\varphi}{2} \cos \theta-R_{1} \cos \theta_{0} \cos \theta\left(1-\cos \left(\varphi_{0}-\varphi\right)\right)-R_{1} \\
& +R_{1}\left(\cos _{0} \cos \theta+\sin _{0} \sin \theta\right) \\
=- & 2 a \sin ^{2} \frac{\varphi_{0}-\varphi}{2} \cos \theta-R_{1}\left(1-\cos \left(\theta_{0}-\theta\right)\right)-2 R_{1} \cos \theta_{0} \cos \theta \sin ^{2} \frac{\varphi_{0}-\varphi}{2} \\
=- & 2 R_{1} \sin ^{2} \frac{\theta_{0}-\theta}{2}-2 \sin ^{2} \frac{\varphi_{0}-\varphi}{2}\left(a \cos \theta+R_{1} \cos \theta_{0} \cos \theta\right) .
\end{array}
$$


Окончательно получаем

$$
\left(\bar{r}_{M M_{0}}, \bar{n}_{M}\right)=-2 R_{1} \sin ^{2} \frac{\theta_{0}-\theta}{2}-2 \sin ^{2} \frac{\varphi_{0}-\varphi}{2} \cos \theta\left(a+R_{1} \cos \theta_{0}\right)
$$

Далее,

$$
\begin{aligned}
\left(\bar{r}_{M M_{0}}, \bar{n}_{M_{0}}\right) & =-\left(\bar{r}_{M_{0} M}, \bar{n}_{M_{0}}\right) \\
& =2 R_{1} \sin ^{2} \frac{\theta_{0}-\theta}{2}+2 \sin ^{2} \frac{\varphi_{0}-\varphi}{2} \cos \theta_{0}\left(a+R_{1} \cos \theta\right)
\end{aligned}
$$

Теперь заметим, что так как

$$
\left\{\begin{array}{l}
\left(a+R_{1} \cos \theta\right) \geqslant\left(a-R_{1}\right)>0 \\
\left(a+R_{1} \cos \theta_{0}\right) \geqslant\left(a-R_{1}\right)>0
\end{array}\right.
$$

то из предыдущих формул и (4.4) получаем для тора

$$
\begin{aligned}
& \frac{\sin ^{2}\left(\left(\theta_{0}-\theta\right) / 2\right)}{r_{M M_{0}}^{2}} \leqslant \frac{1}{4 R_{1}^{2}}, \\
& \frac{\sin ^{2}\left(\left(\varphi_{0}-\varphi\right) / 2\right)}{r_{M M_{0}}^{2}} \leqslant \frac{1}{4\left(a+R_{1} \cos \theta_{0}\right)\left(a+R_{1} \cos \theta\right)} .
\end{aligned}
$$

Поэтому для функции $K_{T, N}\left(M, M_{0}\right)$ на торе из формул $(4.9),(4.12)-(4.15)$ получаем

$$
K_{T, N}\left(M, M_{0}\right)=\frac{1}{r_{M M_{0}}^{3}}+\frac{\lambda_{1}\left(M, M_{0}\right)}{r_{M M_{0}}},
$$

где функция $\lambda_{1}\left(M, M_{0}\right)$ гладкая на $\sigma \backslash M_{0}$ и ограниченная на торе.

Напомним [26; гл. $17, \S 2]$, что если $x=x(U, V), y=y(U, V), z=z(U, V)-$ параметрическое представление поверхности $\sigma$, то для дифференциала площади поверхности справедлива формула

$$
d \sigma=\sqrt{E G-F^{2}} d U d V
$$

где

$$
E=x_{U}^{\prime 2}+y_{U}^{\prime 2}+z_{U}^{\prime 2}, \quad G=x_{V}^{\prime 2}+y_{V}^{\prime 2}+z_{V}^{\prime 2}, \quad F=x_{U}^{\prime} x_{V}^{\prime}+y_{U}^{\prime} y_{V}^{\prime}+z_{U}^{\prime} z_{V}^{\prime}
$$

Поэтому, исходя из формулы (4.2), которая дает параметрическое представление тора, получаем (полагая $U=\theta, V=\varphi$ ):

$$
E=R_{1}^{2}, \quad G=\left(a+R_{1} \cos \theta\right)^{2}, \quad F=0
$$

и окончательно имеем

$$
d \sigma=R_{1}\left(a+R_{1} \cos \theta\right) d \theta d \varphi
$$


Обозначая через $I_{T, N}\left(M_{0}\right)$ интеграл слева в уравнении $(2.6)$, можем теперь записать для тора:

$$
I_{T, N}\left(M_{0}\right)=\frac{1}{4 \pi} \int_{0}^{2 \pi} \int_{0}^{2 \pi} g(M)\left(\frac{1}{r_{M M_{0}}^{3}}+\frac{\lambda_{1}\left(M, M_{0}\right)}{r_{M M_{0}}}\right) R_{1}\left(a+R_{1} \cos \theta\right) d \theta d \varphi
$$

где $0 \leqslant \theta, \varphi \leqslant 2 \pi$.

Аналогично тому, как это было сделано для сферы, введем величину $\widetilde{r}_{M M_{0}}^{2}$ по формуле

$$
\widetilde{r}_{M M_{0}}^{2}=4\left[R_{1}^{2} \sin ^{2} \frac{\theta_{0}-\theta}{2}+\left(a+R_{1} \cos \theta_{0}\right)^{2} \sin ^{2} \frac{\varphi_{0}-\varphi}{2}\right]
$$

и запишем

$$
\frac{1}{r_{M M_{0}}^{3}}=\frac{1}{\widetilde{r}_{M M_{0}}^{3}}+\left(\frac{1}{r_{M M_{0}}^{3}}-\frac{1}{\widetilde{r}_{M M_{0}}^{3}}\right)
$$

Заметим теперь, что из формул (4.4) и (4.21) следует

$$
\begin{aligned}
\widetilde{r}_{M M_{0}}^{2}-r_{M M_{0}}^{2} & =4 \sin ^{2} \frac{\varphi_{0}-\varphi}{2}\left(a+R_{1} \cos \theta_{0}\right) R_{1}\left(\cos \theta_{0}-\cos \theta\right) \\
& =-8 R_{1} \sin ^{2} \frac{\varphi_{0}-\varphi}{2} \sin \frac{\theta_{0}-\theta}{2} \sin \frac{\theta_{0}+\theta}{2}\left(a+R_{1} \cos \theta_{0}\right) .
\end{aligned}
$$

Аналогично формулам (4.14) и (4.15) можно написать

$$
\begin{aligned}
& \frac{\sin ^{2}\left(\left(\theta_{0}-\theta\right) / 2\right)}{\widetilde{r}_{M M_{0}}^{2}} \leqslant \frac{1}{4 R_{1}^{2}}, \\
& \frac{\sin ^{2}\left(\left(\varphi_{0}-\varphi\right) / 2\right)}{\widetilde{r}_{M M_{0}}^{2}} \leqslant \frac{1}{4\left(a+R_{1} \cos \theta_{0}\right)^{2}} \leqslant \frac{1}{4\left(a-R_{1}\right)^{2}}, \\
& \frac{\nu_{1}^{2}}{\nu_{2}^{2}} \geqslant \frac{r_{M M_{0}}^{2}}{\widetilde{r}_{M M_{0}}^{2}}, \quad \frac{\widetilde{r}_{M M_{0}}^{2}}{r_{M M_{0}}^{2}} \geqslant \frac{\nu_{2}^{2}}{\nu_{1}^{2}}
\end{aligned}
$$

где $\nu_{1}=a+R_{1}, \nu_{2}=\min \left(R_{1} ; a-R_{1}\right)$.

Из формул $(4.14),(4.15),(4.24)-(4.26)$ следует, что выражение для $I_{T, N}\left(M_{0}\right)$ можно записать в виде

$$
I_{T, N}\left(M_{0}\right)=\frac{1}{4 \pi} \int_{0}^{2 \pi} \int_{0}^{2 \pi} g(M)\left(\frac{\lambda_{3}^{*}\left(M_{0}\right)}{\widetilde{r}_{M M_{0}}^{3}}+\frac{\lambda_{2}^{*}\left(M, M_{0}\right)}{\widetilde{r}_{M M_{0}}^{2}}+\frac{\lambda_{1}^{*}\left(M, M_{0}\right)}{\widetilde{r}_{M M_{0}}}\right) d \theta d \varphi
$$

для произвольной точки $M_{0}=M\left(x_{0}, y_{0}, z_{0}\right)=M\left(\varphi_{0}, \theta_{0}\right)$, где $\lambda_{3}^{*}\left(M_{0}\right)=R_{1}(a+$ $\left.R_{1} \cos \theta_{0}\right)$, а $\lambda_{2}^{*}\left(M, M_{0}\right)$ и $\lambda_{1}^{*}\left(M, M_{0}\right)$ такие же, как и функции $\lambda_{1}\left(M, M_{0}\right)$ в формуле (4.16).

Перейдем теперь к построению квадратурной формулы для интеграла $I_{T, N}\left(M_{0}\right)$ на торе. Возьмем на осях $O^{*} \varphi$ и $O^{*} \theta$ точки $\varphi_{i}, \theta_{i}=(i-1) h, h=2 \pi /(2 n-1)$, $i=1, \ldots, 2 n$, и точки $\varphi_{0 i}=\varphi_{i}+h / 2, \theta_{0 i}=\theta_{i}+h / 2, i=1, \ldots, 2 n-1$. Тогда линии $\varphi=\varphi_{i}, i=1, \ldots, 2 n$, и $\theta=\theta_{k}, k=1, \ldots, 2 n$, разобьют квадрат $I=[0,2 \pi] \times[0,2 \pi]$ плоскости $O^{*} \varphi \theta$ на прямоугольники $I_{i, k}$ с центрами в точках $M^{*}\left(\varphi_{0 i}, \theta_{0 k}\right), i, k=1, \ldots, 2 n-1$. Образ прямоугольника $I_{i, k}$ на торе $T$ будем обозначать $\sigma_{i, k}$, а образ точки $M^{*}\left(\varphi_{0 i}, \theta_{0 k}\right)$ - через $M_{0 i, 0 k}$. 
ЗАмЕчАниЕ 4.1. Так как точек $\varphi_{0}, \theta_{0 i}$ нечетное число и они являются серединами отрезков $I_{\varphi, i}=\left[\varphi_{i}, \varphi_{i+1}\right], I_{\theta, i}=\left[\theta_{i}, \theta_{i+1}\right], i=1, \ldots, 2 n-1$, то точка $\pi$ является одной из них. Так как интеграл от периодической функции по отрезку, длина которого равна периоду, не зависит от сдвига этого отрезка на любое число, то можно считать, что заданная точка $M^{*}\left(\varphi_{0 j}, \theta_{0 m}\right)$ является серединой квадрата $I$, т.е. имеет координаты $(\pi, \pi)$.

Теперь для интеграла $I_{T, N}\left(M_{0 j, 0 m}\right)$ на торе, как это было сделано для сфферы, рассмотрим сумму

$$
S_{T, N, n}\left(M_{0 j, 0 m}\right)=\sum_{i=1}^{2 n-1} \sum_{k=1}^{2 n-1} g\left(M_{0 i, 0 k}\right) \int_{\sigma_{i, k}} K_{T, N}\left(M, M_{0 j, 0 m}\right) d \sigma_{M}
$$

где $j, m=1, \ldots, 2 n-1$.

Справедлива следуюшая

ТЕОРема 4.1. Пусть функиия $g(M)$ принадлежсит классу $H_{1}(\alpha)$ на торе $T$. Тогда для всех точек $M_{0 j, 0 m}$ выполняется оценка

$$
\left|I_{T, N}\left(M_{0 j, 0 m}\right)-S_{T, N, n}\left(M_{0 j, 0 m}\right)\right| \leqslant C h^{\alpha} \ln n, \quad 0<\alpha \leqslant 1,
$$

әде константа $C$ не зависит от $n$.

ДокАЗАТЕЛьСТво. Используя формулу (4.27), запишем

$$
\begin{aligned}
& \left|I_{T, N}\left(M_{0 j, 0 m}\right)-S_{T, N, n}\left(M_{0 j, 0 m}\right)\right| \\
& \leqslant\left|\lambda_{3}^{*}\left(M_{0 j, 0 m}\right)\right|\left|\sum_{i=1}^{2 n-1} \sum_{k=1}^{2 n-1} \int_{\varphi_{i}}^{\varphi_{i+1}} \int_{\theta_{k}}^{\theta_{k+1}} \frac{\left[g(M)-g\left(M_{0 i, 0 k}\right)\right] d \varphi d \theta}{\widetilde{r}_{M M_{0 j, 0 m}}^{3}}\right| \\
& \quad+\left|\sum_{i=1}^{2 n-1} \sum_{k=1}^{2 n-1} \int_{\varphi_{i}}^{\varphi_{i+1}} \int_{\theta_{k}}^{\theta_{k+1}} \frac{\lambda_{2}^{*}\left(M_{0 j, 0 m}\right)\left[g(M)-g\left(M_{0 i, 0 k}\right)\right] d \varphi d \theta}{\widetilde{r}_{M M_{0 j, 0 m}}^{2}}\right| \\
& \quad+\left|\sum_{i=1}^{2 n-1} \sum_{k=1}^{2 n-1} \int_{\varphi_{i}}^{\varphi_{i+1}} \int_{\theta_{k}}^{\theta_{k+1}} \frac{\lambda_{1}^{*}\left(M_{0 j, 0 m}\right)\left[g(M)-g\left(M_{0 i, 0 k}\right)\right] d \varphi d \theta}{r_{M M_{0 j, 0 m}}}\right| \\
& =\left|\lambda_{3}^{*}\left(M_{0 j, 0 m}\right)\right| I_{T, 1}\left(M_{0 j, 0 m}\right)+I_{T, 2}\left(M_{0 j, 0 m}\right)+I_{T, 3}\left(M_{0 j, 0 m}\right) .
\end{aligned}
$$

По формуле $(2.38)$ на квадрате $I=[0,2 \pi] \times[0,2 \pi]$ в плоскости $O^{*} \varphi \theta$ введем полярные координаты с центром в точке $M^{*}\left(\varphi_{0 j}, \theta_{0 m}\right)$. Тогда в силу замечания 4.1 имеем $0 \leqslant \alpha \leqslant 2 \pi, 0 \leqslant r \leqslant \pi \sqrt{2}$. Теперь аналогично формуле (2.39) можно записать

$$
\widetilde{r}_{M M_{0 j, 0 m}}^{2}=4 r^{2}\left[R_{1}^{2} \frac{\sin ^{2} A}{A^{2}} \frac{\cos ^{2} \alpha}{4}+\left(a+R_{1} \cos \theta_{0}\right)^{2} \frac{\sin ^{2} B}{B^{2}} \frac{\sin ^{2} \alpha}{4}\right]
$$

где $A$ и $B$ определены после формулы (2.39). Из (4.31) в силу соотношения (2.41) получаем

$$
\nu_{1}^{2} \geqslant \frac{\widetilde{r}_{M M_{0 j, 0 m}}^{2}}{r^{2}} \geqslant\left(\nu_{1}^{*}\right)^{2}
$$

где $\nu_{1}$ определено в (4.26), а $\nu_{1}^{*}=\min \left(\frac{4}{3} \pi ; R_{1} ; a-R_{1}\right)>0$. 
Так как по условию теоремы $4.1 g(M) \in H_{1}(\alpha)$, то с учетом неравенств (2.33), (2.37) и неравенств (4.26) получим

$$
I_{T, 3}\left(M_{0 j, 0 m}\right) \leqslant A_{1} h \int_{I} \frac{d \varphi d \theta}{\widetilde{r}_{M M_{0 j, 0 m}}} \leqslant \frac{A_{1}}{\nu_{1}^{*}} h \int_{0}^{2 \pi} d \alpha \int_{0}^{\pi \sqrt{2}} d r=A_{1}^{*} h
$$

где константы $A_{1}, A_{1}^{*}>0$ не зависят от $n$.

Для $I_{T, 2}\left(M_{0 j, 0 m}\right)$ можно написать

$$
\begin{aligned}
I_{T, 2}\left(M_{0 j, 0 m}\right) & \leqslant A_{2} h \int_{I \backslash I_{j, m}} \frac{d \varphi d \theta}{\widetilde{r}_{M M_{0 j, 0 m}}^{2}}+A_{2}^{*} \int_{I_{j, m}} \frac{d \varphi d \theta}{\widetilde{r}_{M M_{0 j, 0 m}}} \\
& =I_{T, 2}^{*}\left(M_{0 j, 0 m}\right)+I_{T, 2}^{* *}\left(M_{0 j, 0 m}\right),
\end{aligned}
$$

где $I_{j, m}=\left[\varphi_{j}, \varphi_{j+1}\right] \times\left[\theta_{m}, \theta_{m+1}\right]$, а константы $A_{2}$ и $A_{2}^{*}$ не зависят от $n$. Переходя к полярным координатам (2.38), получим

$$
\begin{aligned}
& I_{T, 2}^{*}\left(M_{0 j, 0 m}\right) \leqslant \frac{A_{2}}{\left(\nu_{1}^{*}\right)^{2}} h \int_{0}^{2 \pi} d \alpha \int_{h / 2}^{\pi \sqrt{2}} \frac{d r}{r} \leqslant B_{2} h \ln n, \\
& I_{T, 2}^{* *}\left(M_{0 j, 0 m}\right) \leqslant \frac{A_{2}^{*}}{\nu_{1}^{*}} \int_{0}^{2 \pi} d \alpha \int_{0}^{h / \sqrt{2}} d r \leqslant B_{2}^{*} h,
\end{aligned}
$$

где константы $B_{2}$ и $B_{2}^{*}$ не зависят от $n$. Наконец, для $I_{T, 1}\left(M_{0 j, 0 m}\right)$ сделаем преобразования, аналогичные тем, которые были сделаны для $I_{1,1}\left(M_{0 j, 0 m}\right)$ в формуле (2.55). Получим

$$
\begin{aligned}
I_{T, 1}\left(M_{0 j, 0 m}\right) \leqslant & \left|\sum_{i=1}^{2 n-1} \sum_{k=1}^{2 n-1} \int_{I_{i, k}} \frac{\Delta\left(M, M_{0 i, 0 k}\right)}{\widetilde{r}_{M M_{0 j, 0 m}}^{3}} d \varphi d \theta\right| \\
& +\left|\sum_{i=1}^{2 n-1} \sum_{k=1}^{2 n-1} \int_{I_{i, k}} \frac{g_{\varphi}^{\prime}(M)\left(\varphi-\varphi_{0 i}\right)}{\widetilde{r}_{M M_{0 j, 0 m}}^{3}} d \varphi d \theta\right| \\
& +\left|\sum_{i=1}^{2 n-1} \sum_{k=1}^{2 n-1} \int_{I_{i, k}} \frac{g_{\theta}^{\prime}(M)\left(\theta-\theta_{0 k}\right)}{\widetilde{r}_{M M_{0 j, 0 m}}^{3}} d \varphi d \theta\right| \\
= & I_{T, 1}^{*}\left(M_{0 j, 0 m}\right)+I_{T, 1}^{* *}\left(M_{0 j, 0 m}\right)+I_{T, 1}^{* * *}\left(M_{0 j, 0 m}\right) .
\end{aligned}
$$

Как и для $I_{1,1}^{*}\left(M_{0 j, 0 m}\right)$ в $(2.58)$, переходя к полярньп координатам $(2.38)$, получим

$$
I_{T, 1}^{*}\left(M_{0 j, 0 m}\right) \leqslant A_{3} h^{\alpha} .
$$

Для $I_{T, 1}^{* *}\left(M_{0 j, 0 m}\right)$ запишем

$$
\begin{aligned}
I_{T, 1}^{* *}\left(M_{0 j, 0 m}\right) \leqslant & \sum_{i=1}^{2 n-1} \sum_{k=1}^{2 n-1} \int_{I_{i, k}} \frac{\left|g_{\varphi}^{\prime}(M)-g_{\varphi}^{\prime}\left(M_{0 j, 0 m}\right)\right|}{\widetilde{r}_{M M_{0 j, 0 m}}^{3}}\left|\varphi-\varphi_{0 i}\right| d \varphi d \theta \\
& +g_{\varphi}^{\prime}\left(M_{0 j, 0 m}\right)\left|\sum_{i=1}^{2 n-1} \sum_{k=1}^{2 n-1} \int_{I_{i, k}} \frac{\varphi-\varphi_{0 i}}{\widetilde{r}_{M M_{0 j, 0 m}}^{3}} d \varphi d \theta\right|=S_{1}+S_{2}
\end{aligned}
$$


Так как $\left|\varphi-\varphi_{0 i}\right| \leqslant h / 2, g_{\varphi}^{\prime} \in H(\alpha)$ на $I=[0,2 \pi] \times[0,2 \pi]$ и справедливы неравенства (4.26), (4.32), то, переходя к полярным координатам (2.38), получим

$$
\begin{aligned}
S_{1} & \leqslant B_{3} h \int_{I \backslash I_{j, m}} \frac{d \varphi d \theta}{\widetilde{r}_{M M_{0 j, 0 m}}^{3-\alpha}}+B_{3}^{*} \int_{I_{j, m}} \frac{d \varphi d \theta}{\widetilde{r}_{M M_{0 j, 0 m}}^{2-\alpha}} \\
& \leqslant \frac{2 \pi B_{3} h}{\left(\nu_{1}^{*}\right)^{3-\alpha}} \int_{h / 2}^{\pi \sqrt{2}} \frac{d r}{r^{2-\alpha}}+\frac{2 \pi B_{3}^{*}}{\left(\nu_{1}^{*}\right)^{2-\alpha}} \int_{0}^{h / \sqrt{2}} \frac{d r}{r^{1-\alpha}} \\
& \leqslant \widetilde{B}_{3} \frac{h}{h^{1-\alpha}}+\widetilde{B}_{3}^{*} h^{\alpha} \leqslant B_{4} h^{\alpha}
\end{aligned}
$$

Наконец, теперь представляя

$$
\varphi-\varphi_{0 i}=\left(\varphi-\varphi_{0 j}\right)+\left(\varphi_{0 j}-\varphi_{0 i}\right)
$$

и учитывая, что $\varphi_{0 j}=\pi$ и точки $\varphi_{0 i}$ симметричны относительно точки $\varphi_{0 j}$, получаем

$$
S_{2}=0
$$

Таким образом, имеем

$$
I_{T, 1}^{* *}\left(M_{0 j, 0 m}\right) \leqslant B_{4} h^{\alpha}, \quad 0<\alpha \leqslant 1
$$

Оценка, аналогичная оценке (4.42), справедлива и для $I_{T, 1}^{* * *}\left(M_{0 j, 0 m}\right)$.

Проведенные вьше рассуждения доказьвают теорему 4.1.

\section{Список литературы}

1. Мусхелищвили Н. И. Сингулярные интегралњные уравнения. М.: Наука, 1968.

2. Poincare H. Lecons de Mecanique Celeste. V. III. Paris, 1910. X.

3. Hilbert D. Grundziige eine allgemeinen Theorie der linearen Integralgleichungen. LeipzigBerlin, 1912.

4. Гахов Ф. Д. Краевые задачи. М.: Наука, 1977.

5. Лаврентьев M.A. О построении потока, обтекающего дугу заданной формы // Тр. ЦАГИ. 1932. № 118 . С. 3-56.

6. Multhopp H. Die Berechnung der Auftriebsverteilung von Tragfliigeln // Luftfahrtforchung. 1938. V. X. № 4. P. 153-169.

7. Белоцерковский C. M. Тонкая несущая поверхность в дозвуковом потоке газа. М.: Наука, 1965.

8. Белоцерковский C.M., Ништ М.И. Отрывное и безотрывное обтекание тонких крыльев идеальной жидкостью. М.: Наука, 1978.

9. Лифанов И.К., Михайлов А.A. К расчету безотрывного и отрывного обтекания тел // Труды ВВИА им. Н.Е. Жукковского. 1986. № 1313. С. 137-145.

10. Белочерковский С. М., Лифанов И. К., Михайлов А.А. Расчет бесциркуляционного обтекания произвольыых тел // Ученые записки ЦАГИ. 1987. Т. XVIII. № 5. С. 1-10.

11. Belotserkovsky S. M., Lifanov I. K. Method of discrete vortices. Boca Raton: CRC Press, 1993.

12. Панасюк В. В., Саврук М. П., Дацышин А. П. Распределение напряжений около трещин в пластинах и оболочках. Киев: Наукова Думка, 1976.

13. Захаров Е. В., Пименов Ю. В. Численньй анализ дифракции радиоволн. М.: Радио и связь, 1982. 
14. Назарчук З. Т. Численное исследование дифракции волн на цилиндрических структурах. Киев: Наукова Думка, 1989.

15. Белоцерковский С. М., Лифанов И. К. Численные методы в сингулярных интегральных уравнениях. М.: Наука, 1985.

16. Лифанов И.К., Полтавский Л.Н. Пространства дробных отношений, дискретные операторы и их приложения. I // Матем. сб. 1999. Т. 190. №9. С. 41-98.

17. Лифанов И.К., Полтавский Л.Н. Пространства дробных отношений, дискретные операторы и их приложения. II // Матем. сб. 1999. Т. 190. №11. С. 67-134.

18. Адамар Ж. Задача Коши для линейных уравнений с частными производными гиперболического типа. М.: Наука, 1978.

19. Лифанов И. К. Метод сингулярных интегральных уравнений и численный эксперимент. М.: ТОО "Янус", 1995.

20. Тихонов A.H., Самарский A. А. Уравнения математической физики. М.: Наука, 1966.

21. Колтон Д., Кресс Р. Методы интегральных уравнений в теории рассеяния. М.: Мир, 1987.

22. Михлин С. Г. Многомерные сингулярные интегралы и интегральные уравнения. М.: Физматгиз, 1962.

23. Полтавский Л. Н. Математическое обоснование некоторых численных схем в аэродинамике // Дис . . . д докт. физ.-матем. наук. М.: МГУ им. М.В. Ломоносова, 1993.

24. Lifanov I. K., Poltavskii L. N. Quadrature formulae for the Hadamard integral over a curvilinear surface // Russ. J. Numer. Anal. Math. Modelling. 1988. V. 13. № 1. P. 27-44.

25. Смирнов В. И. Курс высшей математики. T. IV, часть вторая. М.: Наука, 1981.

26. Фихтенгольи Г. М. Курс дифференциального и интегрального исчисления. Т. III. М.-Л.: Физматгиз, 1960.

Военный авиационный технический

Поступила в редакцию университет им. Н.Е. Жуковского; 25.12 .2000

Институт вычислительной математики РАН 